\title{
BILLIARD DYNAMICS: AN UPDATED SURVEY WITH THE EMPHASIS ON OPEN PROBLEMS
}

\author{
EUGENE GUTKIN
}

\begin{abstract}
This is an updated and expanded version of our earlier survey article [62]. Section $\S 1$ introduces the subject matter. Sections $\S 2-\S 4$ expose the basic material following the paradigm of elliptic, hyperbolic and parabolic billiard dynamics. In section $\S 5$ we report on the recent work pertaining to the problems and conjectures exposed in the survey 62 . Besides, in section $\S 5$ we formulate a few additional problems and conjectures. The bibliography has been updated and considerably expanded.
\end{abstract}

\section{Contents}

1. Introduction

2. Smooth, strictly convex billiard: elliptic dynamics 4

3. Hyperbolic billiard dynamics 8

4. Polygonal billiard: parabolic dynamics 11

5. Comments, updates, and extensions 16

5.1. The Birkhoff conjecture and related material 16

5.2. The Ivrii conjecture and related material 18

5.3. Extensions of the material in $\S 2 \quad 19$

5.4. Comments and updates for the material in $\S$ 4 20

5.5. Ramifications and extensions of the polygonal billiard 22

5.6. Security for billiard tables and related questions 23

References 25

\section{INTRODUCTION}

Billiard dynamics broadly understood is the geodesic flow on a Riemannian manifold with a boundary. But even this very general framework is not broad enough, e.g., for applications in physics. In these applications the manifold in question is the configuration space of a physical system. Often, it is a manifold with corners and singularities. Some physics models lead to the Finsler billiard [80]: The manifold in question is not Riemannian; it is Finslerian. The simplest examples of Riemannian manifolds with corners

Date: January 14, 2013. 
are plane polygons, and some basic physical models yield the billiard on triangles [28, 82, 50, 60]

The configuration space of the famous gas of elastic balls [129, 137] is structured combinatorially like a euclidean polyhedron of a huge number of dimensions. In fact, this configuration space is much more complicated, because the polyhedron is not flat. The mathematical investigation of this system produced the celebrated Boltzmann Ergodic Hypothesis. After Sinai's seminal papers [129, 130], a modified version of the original conjecture became known as the Boltzmann-Sinai Hypothesis.

However, the bulk of our exposition is restricted to the billiard in a bounded planar domain with piecewise smooth boundary. The reason is threefold. First of all, this setting allows us to avoid lengthy preliminaries and cumbersome formalism: It immediately leads to qualitative mathematical questions. (This was also the opinion of G. D. Birkhoff [12].) Second, there are basic physical models that correspond to planar billiards 60]. Third, and most important, there are fundamental problems on the plane billiard that are still open. The problems are indeed fundamental: They concern the main features of these dynamical systems.

In the body of the paper we introduce several open problems of billiard dynamics. Our choice of the questions is motivated partly by the personal taste and partly by the simplicity of formulation. We review the preliminaries, discuss the motivation, and outline possible angles of attack. We also point out partial results and other evidence toward the answer. Formally, the exposition is self-contained, but the reader may want to consult the literature [102, 86, 107, 138, 26, 57, 60.

For obvious reasons, we will call the planar domain in question the billiard table. Its geometric shape determines the qualitative character of the motion. Historically, three classes of shapes have mostly attracted attention. First, it is the class of smooth and strictly convex billiard tables. For several reasons, the corresponding billiard dynamics is called elliptic. Second, it is the piecewise concave and piecewise smooth billiard tables. The corresponding dynamics is hyperbolic 1 Billiard tables of the third class are the polygons. The corresponding dynamics is parabolic. The three types of the billiard are exposed in $\S 2-\S 3-\S 4$ repectively.

In the rest of the introduction we describe the basic notation and the terminology. Let $Y \subset \mathbb{R}^{2}$ be a compact, connected billiard table. See Figure 1. Its boundary $\partial Y$ is a finite union of $C^{1}$ curves. It may have several connected components. The billiard flow on $Y$ is modelled on the motion of a material point: The "particle" or the "billiard ball". At each time instant, the state of the system is determined by the position of the ball, $y \in Y$, and its velocity, a unit vector $v \in \mathbb{R}^{2}$. (It suffices to consider the motion with the unit speed.) The ball rolls along the ray emanating from $y$, in the direction $v$. At the instant the ball reaches $\partial Y$, its direction changes.

\footnotetext{
${ }^{1}$ There are also convex billiard tables that yield hyperbolic dynamics. See section 3
} 


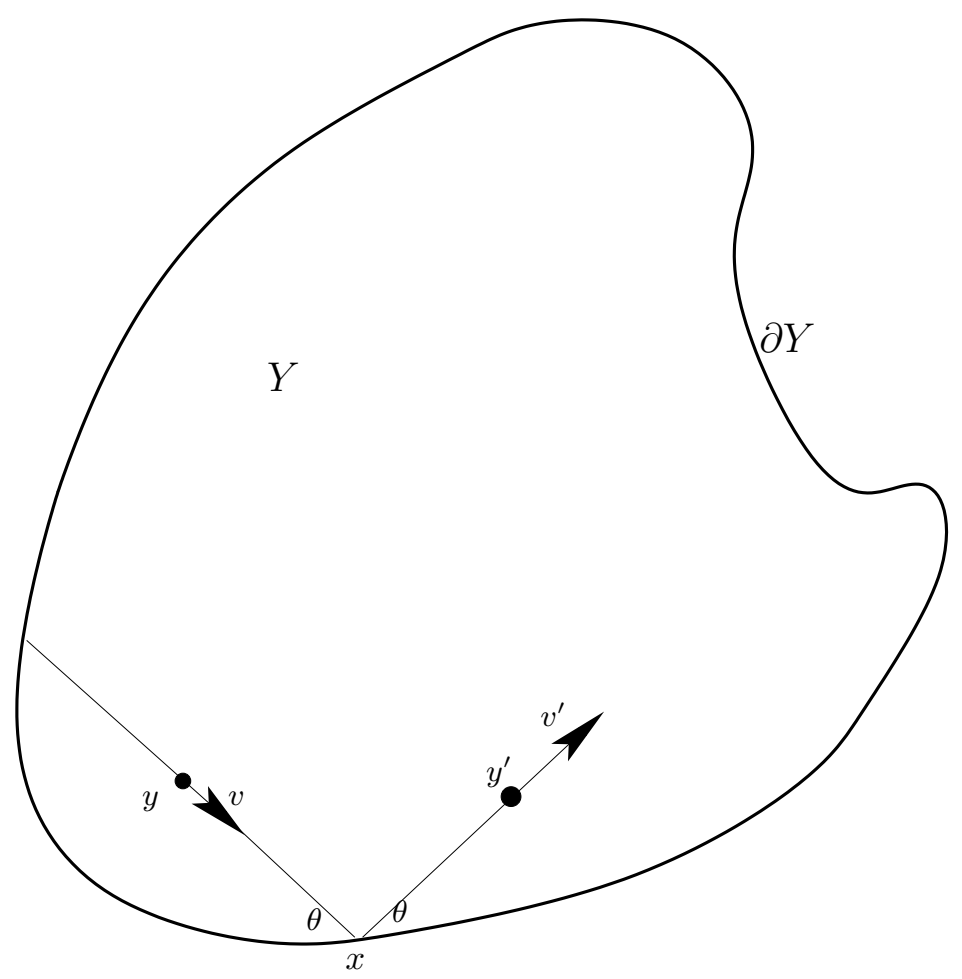

Figure 1. The billiard flow.

Let $x \in \partial Y$ be the point in question, and let $v^{\prime}$ be the new direction. The transformation, $v \mapsto v^{\prime}$, is the orthogonal reflection about the tangent line to $\partial Y$ at $x$. The vector $v^{\prime}$ is directed inward, and the ball keeps rolling.

These rules define: 1) The phase space $\Psi$ of the billiard flow, as the quotient of $Y \times S^{1}$ by the identification $(x, v)=\left(x, v^{\prime}\right)$ above; 2$)$ The billiard flow $T^{t}: \Psi \rightarrow \Psi$. If $Y$ is simply connected, and $\partial Y$ is $C^{1}$, then $\Psi$ is homeomorphic to the three-dimensional sphere 2 In any way, $\operatorname{dim} \Psi=3$, and the reader may think of $\Psi$ as the set of pairs $(y, v)$, such that $v$ is directed inward.

A few remarks are in order. The rules defining the billiard flow stem from the assumptions that the billiard motion is frictionless, and that the boundary of the billiard table is perfectly elastic. The orthogonal reflection rule $v \mapsto v^{\prime}$ insures that billiard orbits are the local minimizers of the distance functional. (This property extends to the Finsler billiard [80].) The reflection rule is not defined at the corners of the boundary. The standard convention is to "stop the ball" when it reaches a corner. Thus, if $\partial Y$ is not $C^{1}$, then there are billiard orbits that are not defined for all times. Their union has zero volume with respect to the Liouville measure defined below.

\footnotetext{
${ }^{2}$ We are not aware of any uses of this observation in the billiard literature.
} 
Set $X=\partial Y$, and endow it with the positive orientation. Choosing a reference point on each connected component, and using the arc length parameter, we identify $X$ with the disjoint union of $k \geq 1$ circles. In this paper, with the exception of $\S \Psi, k=1$. The set $\Phi \subset \Psi$ given by the condition $y \in \partial Y$ is a cross-section for the billiard flow. The Poincaré mapping $\varphi$ : $\Phi \rightarrow \Phi$ is the billiard map and $\Phi$ is its phase space. The terminology is due to G. D. Birkhoff who championed the "billiard ball problem" 12]. Let $x$ be the arc length parameter on $X$. For $(x, v) \in \Phi$ let $\theta$ be the angle between $v$ and the positive tangent to $\partial Y$ at $x$. Then $0 \leq \theta \leq \pi$, where 0 and $\pi$ correspond to the forward and the backward tangential directions respectively. This coordinate system fails at the corners of $\partial Y$. If $\partial Y$ is $C^{1}$, then $\Phi=X \times[0, \pi]$. We will use the notation $\varphi(x, \theta)=\left(x_{1}, \theta_{1}\right)$.

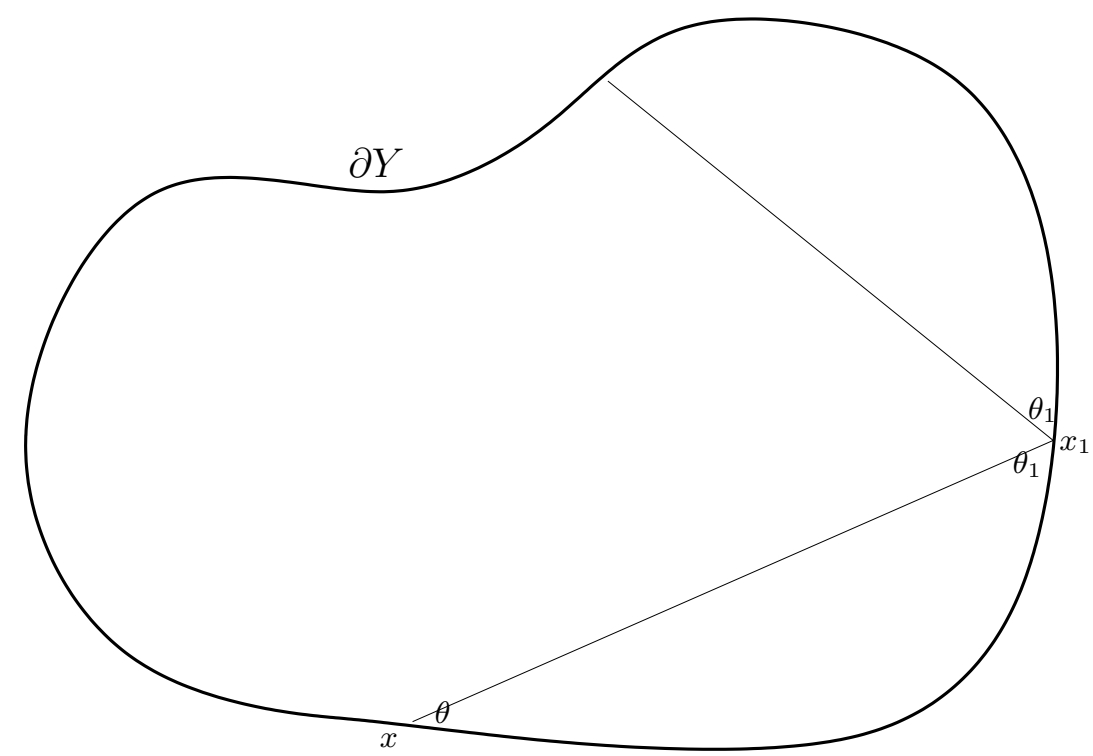

FiguRE 2. The billiard map.

Let $p, q$ be the euclidean coordinates in $\mathbb{R}^{2}$, and let $0 \leq \alpha<2 \pi$ be the angle coordinate on the unit circle. The Liouville measure on $\Psi$ has the density $d \nu=d p d q d \alpha$. It is invariant under the billiard flow. The induced Liouville measure $\mu$ on $\Phi$ is invariant under the billiard map, and has the density $d \mu=\sin \theta d x d \theta$. Both measures are finite. Straightforward computations yield

$$
\nu(\Psi)=2 \pi \operatorname{Area}(Y), \mu(\Phi)=2 \operatorname{Length}(\partial Y) .
$$

\section{Smooth, STRICTLY CONVEX BILLIARD: ELLIPTIC DYNAMICS}

The first deep investigation of this framework is due to G. D. Birkhoff [12]. For this reason, it is often called the Birkhoff billiard. The billiard map is an area preserving twist map [102]. An invariant circle is a $\varphi$-invariant 
curve $\Gamma \subset \Phi$ which is a noncontractible topological circle. Recall that $\Phi$ is a topological annulus. Both components of $\partial \Phi$ are the trivial invariant circles. From the geometric optics viewpoint, $\Phi$ is the space of light rays (i.e., directed lines), and $Y$ is a room whose walls are the perfect mirrors. Then $\Gamma \subset \Phi$ is a one-parameter family of light rays in $Y$, and its envelope $F(\Gamma)$ is the set of focusing points of light rays in this family. Note that $F(\Gamma)$ is not a subset of $Y$, in general. For instance, if $Y$ is an ellipse, then there are invariant curves $\Gamma$ such that $F(\Gamma)$ are confocal hyperbolas.

Let $\Gamma$ be an invariant circle, and let $\gamma=F(\Gamma)$. Then $\gamma \subset \operatorname{Int}(Y)$ [76]. These curves are the caustics of the billiard table. When $\partial Y$ is an ellipse, the caustics are the confocal ellipses. Their union is the region $Y \backslash\left[f f^{\prime}\right]$, where $f, f^{\prime}$ are the foci of $\in Y$. If $Y$ is not a disc, the invariant circles fill out a region, $C(\Phi) \subset \Phi$, whose complement looks like a pair of "eyes". See Figure 3

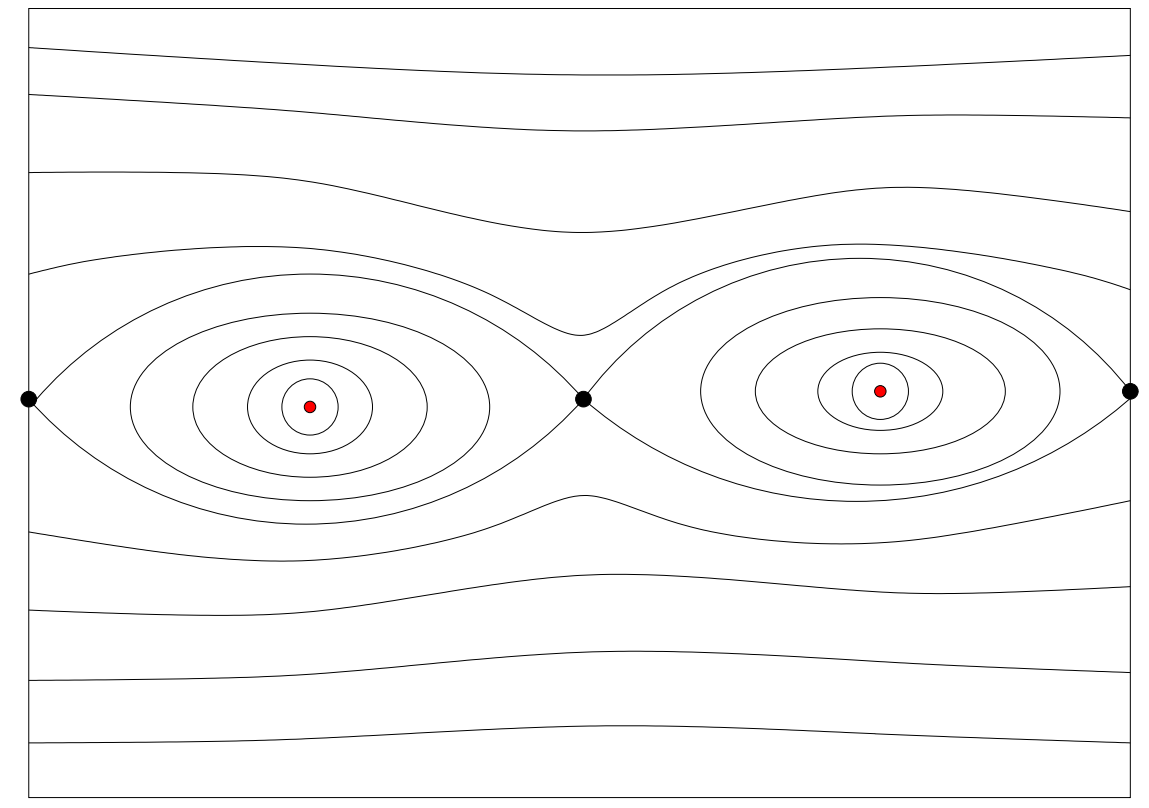

FiguRE 3. The phase space of the billiard map in an ellipse.

Definition 1. A billiard table $Y$ is integrable if the set of invariant circles has nonempty interior.

The most famous open question about caustics is known as the Birkhoff conjecture. It first appeared in print in a paper by Poritsky [121], several years after Birkhoff's death 3

\footnotetext{
${ }^{3}$ In the introduction to 121, the author says that many years ago, when he was a doctoral student of Birkhoff, his advisor communicated the conjecture to him.
} 
Problem 1 (Birkhoff conjecture). Ellipses are the only integrable billiard tables.

A disc is a degenerate ellipse, with $f=f^{\prime}$. The preceding analysis applies, and the invariant circles fill out all of the phase space. M. Bialy proved the converse: If all of $\Phi(Y)$ is foliated by invariant circles, then $Y$ is a disc [10]. See 11 for an extension of this theorem to the surfaces of arbitrary constant curvature. We refer the reader to section $\S 5$ for elaborations and updates on the Birkhoff conjecture.

Let $Y$ be any oval. If $X=\partial Y$ is sufficiently smooth, and its curvature is strictly positive, then the invariant circles fill out a set of positive measure. This was proved by V. Lazutkin under the assumption that $\partial Y$ was of class $C^{333}$ 109]. Lazutkin's proof crucially uses a famous theorem of J. Moser [115] 4] The number 333 is chosen in order to satisfy the assumptions in [115]. The required smoothness was eventually lowered to $C^{6}$ [37]. By a theorem of J. Mather [114, the positive curvature condition is necessary for the existence of caustics.

An invariant noncontractible topological annulus, $\Omega \subset \Phi$, whose interior contains no invariant circles, is a Birkhoff instability region. This is a special case of an important concept for area preserving twist maps [102]. Assume the Birkhoff conjecture, and let $Y$ be a non-elliptical billiard table. Then $\Phi$ contains Birkhoff instability regions. The dynamics in an instability region has positive topological entropy [3]. Hence, the Birkhoff conjecture implies that any non-elliptical billiard has positive topological entropy. By the (metric) entropy of a billiard we will mean the entropy of the Liouville measure. The only examples of convex billiard tables with positive entropy are the Bunimovich stadium [17] and its generalizations. These billiard tables are not strictly convex, and their boundary is only $C^{1}$. The corresponding billiard dynamics is hyperbolic. See $\S 3$. This leads to our next open question.

Problem 2. a) Construct a strictly convex $C^{1}$-smooth billiard table with positive entropy. b) Construct a convex $C^{2}$-smooth billiard table with positive entropy.

Using an ingenuous variational argument, Birkhoff proved the existence of certain periodic billiard orbits 12. His approach extends to area preserving twist maps, and thus yields a more general result on periodic orbits of these dynamical systems 102. In the billiard framework the relevant considerations are especially transparent. A periodic orbit of period $q$ corresponds to an (oriented) closed polygon with $q$ sides, inscribed in $Y$, and satisfying the obvious condition on the angles it makes with $\partial Y$. Birkhoff called these the harmonic polygons. Vice versa, any oriented harmonic $q$-gon $P$ determines

\footnotetext{
${ }^{4}$ This is a seminal paper on the KAM theory. See also [116]. The name KAM stands for Kolmogorov, Arnold and Moser. See [35] for an exposition.
} 
a periodic orbit of period $q$. Let $1 \leq p<q$ be the number of times the pencil tracing $P$ goes around $\partial Y$. The ratio $0<p / q<1$ is the rotation number of a periodic orbit. Fix a pair $1 \leq p<q$, with $p$ and $q$ relatively prime. Let $X(p, q)$ be the set of all inscribed $q$-gons that go $p$ times around $\partial Y$. The space $X(p, q)$ is a manifold with corners. For $P \in X(p, q)$ let $f(P)$ be the circumference of $P$. Then harmonic polygons are the critical points of the function $f: X(p, q) \rightarrow \mathbb{R}$. Birkhoff proved that $f$ has at least two distinct critical points. One of them delivers the maximum, and the other a minimax to the circumference. The corresponding periodic billiard orbits are the Birkhoff periodic orbits with the rotation number $p / q$.

By way of example, we take the rotation number $1 / 2$. Then the maximal Birkhoff orbit yields the diameter of $Y$. The minimax orbit corresponds to the width of $Y$. When the diameter and the width of $Y$ are equal, the boundary $\partial Y$ is a curve of constant width; then we have a one-parameter family of periodic orbits with the rotation number $1 / 2$. They fill out the "equator" of $\Phi$. There are other examples of ovals with one-parameter families of periodic orbits having the same length and the same rotation number. See [94] and [59, 68] for different approaches.

One of the basic characteristics of a dynamical system is the growth rate of the number of periodic points. In order to talk about it, we need a counting function. The standard counting function $f_{Y}(n)$ for the billiard map is the number of periodic points of the period at $\operatorname{most} n$. (See $\S 3$ and $\S 4$ for other examples.) The set of periodic points is partitioned into periodic orbits, and let $F_{Y}(n)$ be the number of periodic orbits of period at most $n$. Birkhoff's theorem bounds $F_{Y}(n)$ from below by the number of relatively prime pairs $1 \leq p<q \leq n$. This implies a universal cubic lower bound $f_{Y}(n) \geq c n^{3}$. See, e. g., 85 .

Since an oval may have infinitely many periodic points of the same period, there is no universal upper bound on $f_{Y}(n)$. The size of a measurable set is naturally estimated by its measure. Let $\mathcal{P} \subset \Phi$ (resp. $\left.\mathcal{P}_{n} \subset \Phi\right)$ be the set of periodic points (resp. periodic points of period $n$ ). For example, if $Y$ is a table of constant width, then $\mathcal{P}_{2} \subset \Phi$ is the equator. Although it is infinite, $\mu\left(\mathcal{P}_{2}\right)=0$. Since $\mathcal{P}=\cup_{n=2}^{\infty} \mathcal{P}_{n}$, a disjoint union, $\mu(\mathcal{P})=\sum_{n=2}^{\infty} \mu\left(\mathcal{P}_{n}\right)$. Thus, $\mu(\mathcal{P})=0$ iff $\mu\left(\mathcal{P}_{n}\right)=0$ for all $n=2,3,4, \ldots$

The famous Weyl formula gives the leading term and the error estimate for the spectral asymptotics of the Laplace operator (with either Dirichlet or Neumann boundary conditions) in a bounded domain of the euclidean space (of any number of dimensions). The (also famous) Weyl conjecture predicts the second term of the asymptotic series [148]. A theorem of $\mathrm{V}$. Ivrii [95] establishes the Weyl conjecture for a euclidean domain under the assumption that the set of periodic billiard orbits has measure zero 5

\footnotetext{
${ }^{5} \mathrm{~A}$ more general formula for the spectral asymptotics of the Laplacean, due to Safarov and Vassiliev, contains a term accounting for periodic orbits [124. If periodic points yield a set of measure zero, this term vanishes.
} 
Ivrii conjectured that the assumption $\mu(\mathcal{P})=0$ was superfluous: It should hold for any euclidean domain with a smooth boundary. Members of the Sinai's dynamics seminar in Moscow promised to him in 1980 to prove the desideratum in a few days ... The question is still open. Problem 3 below states the conjecture for plane domains.

Problem 3 (Ivrii conjecture). Let $Y$ be a piecewise smooth billiard table. i) Prove that $\mu(\mathcal{P})=0$. ii) Prove that $\mu\left(\mathcal{P}_{n}\right)=0$ for all $n$.

Although Problem 3 concerns arbitrary billiard tables, it is especially challenging for the Birkhoff billiard, hence we have put the problem into this section. It is convenient to designate by, say, $I_{n}$ the claim $\mu\left(\mathcal{P}_{n}\right)=0$. Thus, Ivrii conjecture amounts to proving $I_{n}$ for all $n \geq 2$. Claim $I_{2}$ is obvious. Proposition $I_{3}$ is a theorem of M. Rychlik [123]. His proof depends on a formal identity, verified using Maple. L. Stojanov simplified the proof, and eliminated the computer verification 135. Ya. Vorobets gave an independent proof [146]. His argument applies to higher dimensional billiards as well. M. Wojtkowski [152] obtained Rychlik's theorem as an application of the mirror equation of the geometric optics and the isoperimetric inequality. See [76] for other applications.

Ivrii's conjecture is known to hold in many special cases, e.g., for hyperbolic and parabolic billiard tables. See $\S 3$ and $\S$ 4. It holds for billiard tables with real analytic boundary [124. For the generic billiard table the sets $\mathcal{P}_{n}$ are finite for all $n$ [119]. The billiard map for a Birkhoff billiard table is an area preserving twist map. However, there are smooth area preserving twist maps such that $\mu(\mathcal{P})>0$. Thus, Ivrii's conjecture is really about the billiard map!

Recently Glutsyuk and Kudryashov announced a proof of Proposition $I_{4}$ [51. See Section 5.3 for further comments.

\section{HyPERBOLIC BILLIARD DYNAMICS}

It is customary to say that a billiard table is hyperbolic if the associated dynamics is hyperbolic. The dynamics in question may be the billiard flow or the billiard map or the induced map on a subset of the phase space. For concreteness, we will call a billiard table hyperbolic if the corresponding billiard map is hyperbolic. The modern approach to hyperbolic dynamics crucially uses the Oseledets multiplicative ergodic theorem [117. See [102, 86] for a general introduction into the hyperbolic dynamics and [103, 18, 138, (resp. [26]) for introductory (resp. thorough) expositions of the hyperbolic billiard.

The first hyperbolic billiard tables were made from concave arcs. As a motivation, let us consider the following construction. Let $P$ be a convex polygon. Replace some of the sides of $P$ by circular arcs whose centers are 
sufficiently far from $P$. The result is a "curvilinear polygon", $Y$, approximating $P$. Choosing appropriate center points, we insure that the "curved sides" of $Y$ are convex inward. It is not important that they be circular, as long as they are smooth and convex inward.

This class of billiard tables arose in the work of Ya. Sinai on the BoltzmannSinai gas [129]6 In the Boltzmann gas the identical round molecules are confined by a box. Sinai has replaced the box by periodic boundary conditions. Thus, the molecules of the Boltzmann-Sinai gas move on a flat torus. In the "real world", the confining box is three-dimensional and the number of moving molecules is enormous. In the Sinai "mathematical caricature", there are only two molecules on a two-torus. The system reduces to the geodesic flow on a flat torus with a round hole. Represent the flat torus by the $2 \times 2$ square, so that the hole is the central disc of radius $1 / 2$. By the fourfold symmetry, the problem reduces to the billiard on the unit square with the deleted quarter-disc of radius 1/2, centered at a vertex. See Figure 4 .

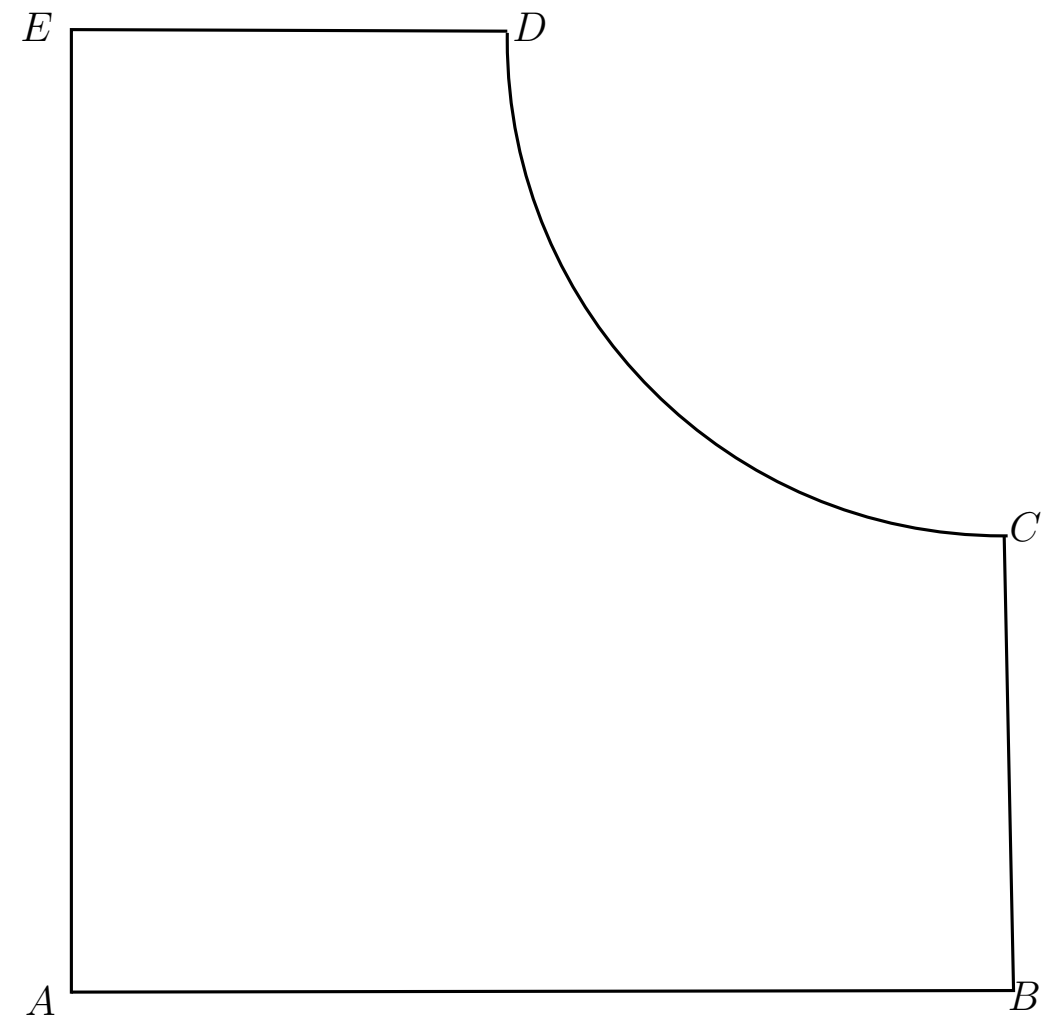

Figure 4. The Sinai billiard table.

\footnotetext{
${ }^{6}$ See the Appendix by D. Szasz in [137].
} 
This domain is known as the Sinai billiard $\square$ Let now $P$ be a (not necessarily convex) $n$-gon. Let $Y$ be the region obtained by replacing $1 \leq m<n$ (resp. all) of the sides of $P$ by circular arcs, satisfying the conditions above. Then $Y$ is a semi-dispersive (resp. dispersive) billiard table. The circular arcs (resp. the segments) of $\partial Y$ are its dispersive (resp. neutral) components. This terminology extends to the billiard tables whose dispersive boundary components are smooth, convex inward curves. These are the (semi)dispersive billiard tables. The table in Figure 4 has one dispersive and four neutral boundary components.

In [130] Sinai proved the hyperbolicity of dispersive billiard tables. After the discovery by L. Bunimovich that the stadium and similar billiard tables are hyperbolic [17, mathematicians started searching for geometric criteria of hyperbolicity. The notion of an invariant cone field [149, 101] proved to be very useful.

Denote by $V_{z}$ the tangent plane to the phase space at $z \in \Phi$. The differential $\varphi_{*}$ is a linear map from $V_{z}$ to $V_{\varphi(z)}$. By our convention, a subset of a vector space is a cone if it is invariant under multiplications by all scalars.

Definition 2. A family $\mathcal{C}=\left\{C_{z} \subset V_{z}: z \in \Phi\right\}$ is an invariant cone field if the following conditions are satisfied.

- 1. The closed cone $C_{z}$ is defined for almost all $z \in \Phi$, and the map $z \mapsto C_{z}$ is measurable.

- 2. The cone $C_{z}$ is has nonempty interior.

- 3. We have $\varphi_{*}\left(C_{z}\right) \subset C_{\varphi(z)}$.

- 4. There exists $n=n(z)$ such that $\varphi_{*}^{n}\left(C_{z}\right) \subset \operatorname{int}\left(C_{\varphi^{n}(z)}\right)$.

The hyperbolicity is equivalent to the existence of an invariant cone field [149. Wojtkowski constructed invariant cone fields for several classes of billiard tables [150]. In addition to the dispersive tables and the generalized stadia, he found invariant cone fields for a wide class of locally strictly convex tables. Wojtkowski's approach was further extended by Bunimovich, V. Donnay, and R. Markarian [26]. Using these ideas, B. Gutkin, U. Smilanski and the author constructed hyperbolic billiard tables on surfaces of arbitrary constant curvature [53].

Problem 4. Is every semi-dispersive billiard table hyperbolic?

Let $Y$ be a semi-dispersive $n$-gon with only one neutral component. Let $Y^{\prime}$ be the reflection of $Y$ about this side, and set $Z=Y \cup Y^{\prime}$. Since $Z$ is

\footnotetext{
${ }^{7}$ Unfortunately, there is a fair amount of confusing terminology in the literature. Mathematicians often use the expressions like "Sinai's billiard" or "the Sinai table" or "a dispersive billiard" interchangeably. Physicists tend to mean by "the Sinai billiard" a special billiard table, although not necessarily that of Figure 4.
} 
a dispersive billiard table, it is hyperbolic. By the reflection symmetry, the table $Y$ is also hyperbolic. In special cases, the reflection trick yields the hyperbolicity of semi-dispersive $n$-gons with $m<n-1$ dispersive components. For instance, let $P$ be a triangle with an angle $\pi / n$. Let $Y$ be the semidispersive triangle, whose only dispersive component is located opposite the $\pi / n$ angle. Reflecting $Y$ successively $2 n$ times, we obtain a dispersive billiard table, $Z$. Thus, $Z$ is hyperbolic. By the symmetry, the table $Y$ is hyperbolic as well. A suitable generalization of the reflection trick will work if $P$ is a rational polygon. See $\S$. The special case $m=1$ of Problem 4 is closely related to Problem 9 of $\S 4$.

Dispersive billiard tables are ergodic [20]. There are examples of hyperbolic, but nonergodic billiard tables [150]. The consensus is that a typical hyperbolic billiard is ergodic. For instance, the stadium and its relatives are ergodic [136]. There are no examples of strictly convex hyperbolic billiard tables. See Problem 2.

For the rest of this section, we consider only dispersive billiard tables. Referring the reader to [19, 20, 24, 137, 25, 153] for a discussion of their chaotic properties and to open questions about, e.g., the decay of correlations, we concentrate on the statistics of periodic orbits in hyperbolic billiards. The set of periodic points of any period is finite; let $f_{Y}(n)$ be the number of periodic points, whose period is less than or equal to $n$. The asymptotics of $f_{Y}(n)$, as $n \rightarrow \infty$, is an important dynamical characteristic. By theorems of Stojanov and Chernov [134, 19], there are $0<h_{-}<h_{+}<\infty$ such that

$$
0<h_{-} \leq \liminf _{n \rightarrow \infty} \frac{\log f_{Y}(n)}{n} \leq \limsup _{n \rightarrow \infty} \frac{\log f_{Y}(n)}{n} \leq h_{+}<\infty .
$$

The following two problems were contributed by N. Chernov.

Problem 5. Does the limit

$$
h=\lim _{n \rightarrow \infty} \frac{\log f_{Y}(n)}{n}
$$

exist?

Problem 6. If the limit in equation (3) exists, is $0<h<\infty$ the topological entropy of the billiard map?

Problems 5 and 6 fit into the general relationship between the distribution of periodic points and the topological entropy 98. However, the singularities, which constitute the paramount feature of billiard dynamics, preclude the applicability of smooth ergodic theory. Other techniques have to be developed [23, 70, 71].

\section{Polygonal BILliard: PARABOLIC DYNAMiCS}

The polygon $P$ that serves as a billiard table is not required to be convex or simply connected. It may also have barriers, i. e., obstacles without 
interior. It is rational if the angles between its sides are of the form $\pi m / n$. Let $N=N(P)$ be the least common denominator of these rational numbers. A classical construction associates with $P$ a closed surface $S=S(P)$ tiled by $2 N$ copies of $P$. The surface $S$ has a finite number of cone points; the cone angles are integer multiples of $2 \pi$. Suppose that $P$ is a simple polygon 8 and let $m_{i} \pi / n_{i}, 1 \leq i \leq p$, be its angles. The genus of $S(P)$ satisfies [57]

$$
g(S(P))=1+\frac{N}{2} \sum_{i=1}^{p} \frac{m_{i}-1}{n_{i}} .
$$

Equation (4) implies that $S(P)$ is a torus if and only if $P$ tiles the plane under reflections. The billiard in $P$ is essentially equivalent to the geodesic flow on $S(P)$. This observation was first exploited by A. Katok and A. Zemlyakov [104, and $S(P)$ is often called the "Katok-Zemlyakov surface". However, the construction has been in the literature (at least) since the early 20-th century [133, 45]. We refer to the surveys [57, 60, 131, 113, 138, for extensive background material.

Surfaces $S(P)$ are examples of translation surfaces, which are of independent interest [74. From the viewpoint of classical analysis, a translation surface is a closed Riemann surface with a holomorphic linear differential. Using holomorphic quadratic (as opposed to linear) differentials, we arrive at the notion of half-translation surfaces [73, 74. Billiard orbits on a polygon become geodesics on the corresponding translation (or the half-translation) surface. Since billiard orbits change directions at every reflection, the notion of the direction of an orbit is not well defined. Geodesics on a translation surface, on the contrary, do not change their directions. This yields a technical advantage of translation surfaces over polygons [104. The crucial advantage comes, however, from the natural action of the group $\operatorname{SL}(2, \mathbb{R})$ on translation surfaces [106, 110, 111, 144, 131]. See section 5 for elaborations.

The geodesic flow of any translation surface, $S$, decomposes into the oneparameter family of directional flows $b_{\theta}^{t}, 0 \leq \theta<2 \pi$. The flow $b_{\theta}^{t}$ is identified with the linear flow on $S$ in direction $\theta$. The Lebesgue measure on $S$ is preserved by every $b_{\theta}^{t}$. Thus, not only is the billiard flow of a rational polygon not ergodic, it decomposes as a one-parameter family of directional billiard flows. Let $S$ be an arbitrary translation surface. A theorem of Kerckhoff, Masur, and Smillie [106] says that the flows $b_{\theta}^{t}$ are uniquely ergodic for Lebesgue almost all $\theta$. In particular, the directional billiard flow of a rational polygon is ergodic for almost every direction. The set $\mathcal{N}(S) \subset[0,2 \pi)$ of non-uniquely ergodic directions has positive Hausdorff dimension for the typical translation surface [112]; for particular classes of rational polygons and translation surfaces the sets $\mathcal{N}(S)$ are countably infinite [55, 144, 27]. We point out that a typical translation surface does not correspond to any polygon, which illustrates the limitations of this relationship for the study of polygonal billiard. See section 5 for elaborations on the polygonal billiard

\footnotetext{
${ }^{8}$ A polygon $P$ is simple if $\partial P$ is connected.
} 
and translation surfaces.

Much less is known about the billiard in irrational (i. e., arbitrary) polygons. Denote by $\mathcal{T}(n)$ the moduli space of simple euclidean $n$-gons. Since the billiard dynamics is invariant under scaling, in $\mathcal{T}(n)$ we identify polygons that coincide up to scaling. The space $\mathcal{T}(n)$ is a finite union of components which correspond to particular combinatorial data. We will refer to them as the combinatorial type components. Each component is homeomorphic to a relatively compact set of the maximal dimension in a Euclidean space. Let $\lambda$ be the probability measure on $\mathcal{T}(n)$, such that its restrictions to the combinatorial type components are the corresponding Lebesgue measures. For instance, the space $\mathcal{T}(3) \subset \mathbb{R}^{2}$ is given by $\mathcal{T}(3)=\{(\alpha, \beta): 0<\alpha \leq \beta<\pi / 2\}$. Thus, $\mathcal{T}(3)$ itself is a plane triangle. By a theorem in [106], the set $\mathcal{E}(n) \subset \mathcal{T}(n)$ of ergodic $n$-gons is residual in the sense of Baire category [118.

Problem 7. Is $\lambda(\mathcal{E}(n))>0$ ?

The case of $n=3$ is especially interesting, since the mechanical system of three elastic point masses moving on a circle (see Figure 5) leads to the billiard in an acute triangle [50, 22. Let $m_{1}, m_{2}, m_{3}$ be the masses. Then the angles of the corresponding triangle $\Delta\left(m_{1}, m_{2}, m_{3}\right)$ satisfy

$$
\tan \alpha_{i}=m_{i} \sqrt{\frac{m_{1}+m_{2}+m_{3}}{m_{1} m_{2} m_{3}}} .
$$

We point out that the rationality of the triangle corresponding to a mechanical system of point masses does not have any obvious physical meaning. In the limit, when $m_{3} \rightarrow \infty$, we obtain the physical system of two elastic particles on an interval. The limit of $\Delta\left(m_{1}, m_{2}, m_{3}\right)$ is the right triangle whose angles satisfy $\tan \alpha_{1}=\sqrt{m_{1} / m_{2}}, \tan \alpha_{2}=\sqrt{m_{2} / m_{1}}$.

Let $P$ be an irrational polygon. Let $\alpha_{1}, \ldots, \alpha_{k}$ be its angles. If the numbers $\alpha_{i} / \pi$ simultaneously admit a certain super-exponentially fast rational approximation, then $P$ is ergodic [147]. This remarkable theorem yields explicit examples of ergodic polygons. However, it does not help with the above problem. There is some numerical evidence that irrational polygons are ergodic and have other stochastic properties [4, 22. So far, there are no theorems confirming or precluding this.

Problem 8. Give an example of an irrational but nonergodic polygon.

Let $P$ be an arbitrary $n$-gon, and let $a_{1}, \ldots, a_{n}$ be its sides. For $1 \leq$ $i \leq n$ let $\Phi_{i} \subset \Phi$ be the set of elements whose base points belong to the side $a_{i}$. Then $\Phi=\cup_{i=1}^{n} \Phi_{i}$, a disjoint decomposition. By equation (11), $\mu\left(\Phi_{i}\right)=2 \operatorname{Length}\left(a_{i}\right)$. The next question/conjecture concerns the structure of invariant sets in the phase space of a nonergodic polygon. (Compare with 


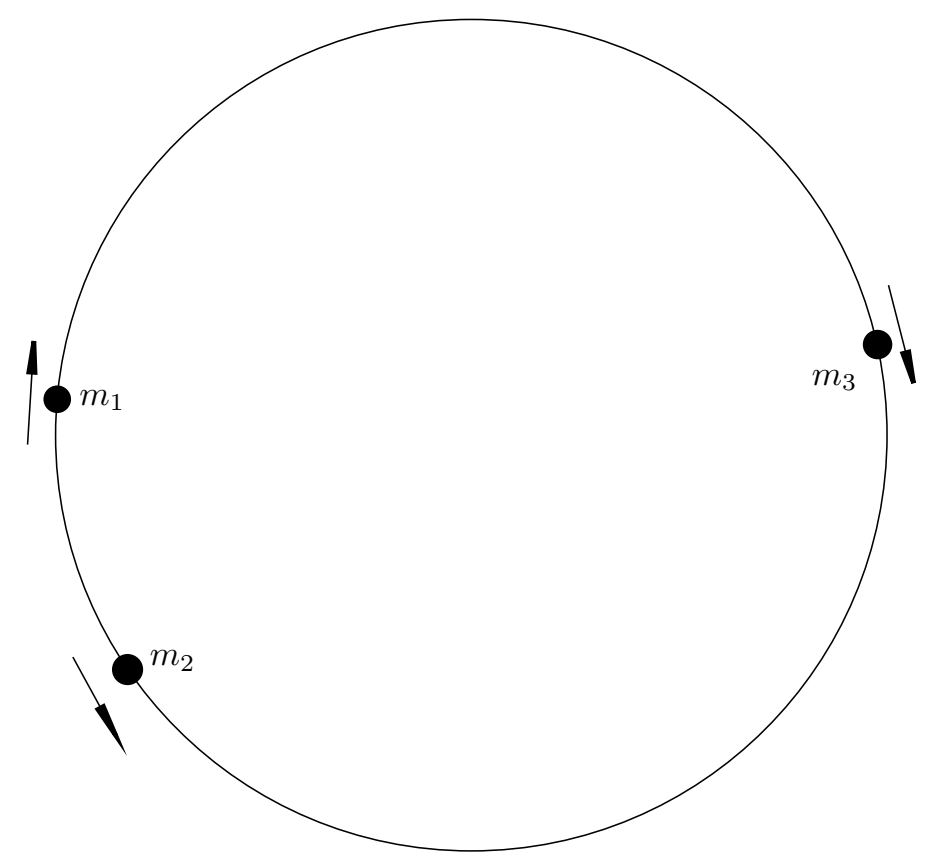

FiguRE 5. Three perfectely elastic particles on the circle.

Problem 4 in $\S$ 3.) By [106, the conjecture holds for rational polygons.

Problem/Conjecture 9. Let $P$ be an irrational $n$-gon, and let $M \subset \Phi$ be an invariant set of positive measure. If $\Phi_{i} \subset M$ for some $1 \leq i \leq n$, then $M=\Phi$.

The subject of periodic billiard orbits in polygons requires only elementary euclidean geometry, and has immediate applications to physics. For instance, let $\Delta\left(m_{1}, m_{2}, m_{3}\right)$ be the acute triangle corresponding to the system of three elastic point masses equation (5). Periodic billiard orbits in $\Delta\left(m_{1}, m_{2}, m_{3}\right)$ correspond to the periodic motions of this mechanical system. 9 Ironically, periodic orbits in polygons turned out to be especially elusive.

Problem 10. Does every polygon have a periodic orbit?

Every rational polygon has periodic orbits, and much is known about them. Certain classes of irrational polygons have periodic orbits [28, 82]. Every acute triangle has a classical periodic orbit - the Fagnano orbit 61. It corresponds to the inscribed triangle of minimal perimeter. It is not

\footnotetext{
${ }^{9}$ Another connection between billiards and physics arises in the study of mechanical linkages 132 .
} 
known if every acute triangle has other periodic orbits; it is also not known if every obtuse triangle has a periodic orbit [47, 83]. See $\S[5$ for updates and elaborations.

A periodic orbit with an even number of segments is contained in a parallel band of periodic orbits of the same length. The boundary components of a band are concatenations of singular orbits, the so-called generalized diagonals [99. These are the billiard orbits with endpoints at the corners. Periodic orbits with an odd number of segments (e. g., the Fagnano orbit) are isolated. They seem to be rare; a rational polygon has at most a finite number of them. Denote by $f_{P}(\ell)$ the number of periodic bands of length at most $\ell$. This counting function for periodic billiard orbits in polygons grows subexponentially $[99,70,71$. Conjecturally, there should be a universal polynomial upper bound on $f_{P}(\cdot)$. See section 5 for elaborations.

Problem 11. Find efficient upper and lower bounds on $f_{P}$ for irrational polygons.

From now until the end of this section we consider only rational polygons. By results of Masur [110, 111 and Boshernitzan [15, 16, there exist numbers $0<c_{*}(P) \leq c^{*}(P)<\infty$ such that $c_{*}(P) \ell^{2} \leq f_{P}(\ell) \leq c^{*}(P) \ell^{2}$ for sufficiently large $\ell$. We will refer to these inequalities as the quadratic bounds on periodic billiard orbits. The numbers $c_{*}(P), c^{*}(P)$ are the quadratic constants.

Problem 12. Find efficient estimates for quadratic constants.

In all known examples $f_{P}(\ell) / \ell^{2}$ has a limit, i. e., $c_{*}(P)=c^{*}(P)=c(P)$. In this case, we say that the polygon has quadratic asymptotics. The preceding definitions and questions have obvious counterparts for translation surfaces, where periodic billiard orbits are replaced by closed geodesics. There is a special class of polygons and surfaces: Those satisfying the lattice property, or, simply, the lattice polygons and translation surfaces [143, 73, 74. They have quadratic asymptotics, and there are general expressions for their quadratic constants [55, 143, 145, 73, 74]. Besides, the quadratic constants for several lattice polygons are explicitly known [143, 145. These formulas contain rather subtle arithmetic. The (normalized) quadratic constant of any lattice polygon is an algebraic number [74.

There is a lot of information about lattice polygons and lattice translation surfaces. Lattice polygons seem to be very rare. All acute lattice triangles have been determined [105, 122]. For obtuse triangles the question is still open. There is a $\mathrm{SL}(2, \mathbb{R})$-invariant Lebesgue-class finite measure on the strata of the moduli space $\mathcal{M}_{g}$ of translation surface of fixed genus [39]. The strata correspond to the partitions $2 g-2=p_{1}+\cdots+p_{t}: p_{i} \in \mathbb{N}$. Hence, we can speak of a generic translation surface in $\mathcal{M}_{g}\left(p_{1}, \ldots, p_{t}\right)$. The quadratic constant of the generic translation surface $S \in \mathcal{M}_{g}\left(p_{1}, \ldots, p_{t}\right)$ depends only on the stratum [39, 42, 41]. These results crucially use the relationship 
between the Teichmüller flow 93] on $\mathcal{M}_{g}$ and linear flows on translation surfaces. See the surveys [113, 36, 5] for this material.

The results for generic translation surfaces have no consequences for rational polygons, since the generic translation surface does not correspond to a polygon. However, an extension of the Teichmüller flow approach establishes the quadratic asymptotics for a special, but nontrivial class of rational polygons [40].

Problem 13. Does every rational polygon have quadratic asymptotics?

The following section contains updates, elaborations, and extensions of the preceding material.

\section{Comments, updates, And extensions}

At the time of writing this text, the problems discussed in the survey 62 ] remain open. However, the works that have since appeared contain substantial relevant material. The main purpose of this section is to comment on this material. At the same time, we take the opportunity to add a few extensions and ramifications that for some reasons did not appear in the survey 62. Accordingly, we have updated and expanded the bibliography.

A few recent books contain discussions of the billiard ball problem. The book [26] is a thorough exposition of the hyperbolic billiard dynamics. See $\S$ 3. The treatise 9] contains several discussions of connections between the billiard ball problem and geometry. The material in 9] is relevant for all three types of billiard tables discussed above. The latter can be also said about [139]. However, the two books are fundamentally different. The book 9] is a comprehensive treatise on geometry, where the billiard ball problem is but one of a multitude of illustrative examples and applications, while [139. is addressed primarily to young American students, and discusses a few instances pertaining to the geometric aspect of the billiard. The book [52] exposes several applications of convex geometry to the billiard ball problem. See the material in $\S 2$.

\subsection{The Birkhoff conjecture and related material.}

The traditional formulation of the Birkhoff integrability conjecture is in terms of the billiard map. See Definition 1 and Problem 1. The billiard flow on a smooth, convex table $Y$ is a Hamiltonian system with 2 degrees of freedom. The Hamiltonian version of Problem 1 is as follows:

Problem 1a. Let $Y$ be a smooth, convex billiard table. If the billiard flow on $Y$ is an integrable Hamiltonian system, then $Y$ is an ellipse.

Although Problem 1 and Problem 1a are obviously related, a resolution

of either one of them would not directly imply a resolution of the other. The 
results of S. Bolotin [14 provide evidence supporting a positive resolution of Problem 1a.

Let $\gamma \subset \mathbb{R}^{2}$ be a closed convex curve. Denote by $|\gamma|$ its perimeter. The string construction associates with any $\ell>|\gamma|$ a closed convex curve $G(\gamma, \ell)$ containing $\gamma$ in its interior. The curve $G(\gamma, \ell)$ is obtained by the following "physical process". We take a ring of length $\ell$ made from a soft, nonstretchable material and wrap it around $\gamma$. We pull the ring tight with a pencil; then, holding it tight, we rotate the pencil all the way around $\gamma$. The moving pencil will then trace the curve $G(\gamma, \ell)$. A gardener could use this process to design fences around his flower beds. For this reason, the procedure is sometimes called the gardener construction [9].

Let $Y=Y(\gamma, \ell) \subset \mathbb{R}^{2}$ be the billiard table whose boundary is $G(\gamma, \ell)$. Then $\gamma$ is a caustic for the billiard on $Y$ [109, 76, 139]. If $\gamma_{1}$ is an ellipse and $\ell_{1}>\left|\gamma_{1}\right|$, then $\gamma_{2}=G\left(\gamma_{1}, \ell_{1}\right)$ is a confocal ellipse. Let now $\ell_{2}>\left|\gamma_{2}\right|$. Then $\gamma_{3}=G\left(\gamma_{2}, \ell_{2}\right)$ is a confocal ellipse containing $\gamma_{1}$. There is a unique $\ell_{3}$ such that $\gamma_{3}=G\left(\gamma_{1}, \ell_{3}\right)$. This transitivity property of gardener's construction is a consequence of the integrability of billiard on an ellipse. The Birkhoff conjecture suggests the following problem.

Problem 1b. Ellipses are the only closed convex curves satisfying the above transitivity.

This entirely geometric variant of the Birkhoff conjecture is due to $\mathrm{R}$. Melrose. A positive solution of Problem $1 \mathrm{~b}$ is the subject of the $\mathrm{PhD}$ thesis of Melrose's student E. Amiran [2]. However, the work [2] contains a serious gap, and the question remains open.

Let now $\gamma$ be any closed convex curve. For $\ell>|\gamma|$ let $Y(\ell)=Y(\gamma, \ell)$ be the corresponding family of convex billiard tables, and let $0<\rho_{\gamma}(\ell)<1 / 2$ be the rotation number of the caustic $\gamma \subset Y(\ell)$. The rotation function $\rho(\ell)=\rho_{\gamma}(\ell)$ is continuous and monotonically increasing, but not strictly, in general. Let $r \in(0,1 / 2)$ be such that $\rho^{-1}(r)=[a(r), b(r)]$ is a nontrivial interval. Then i) $r$ is rational; ii) for $\ell \in[a(r), b(r)]$ the billiard map of $Y(\ell)$ restricted to the caustic $\gamma$ is not a rotation. The converse also holds; $[a(r), b(r)]$ are the phase locking intervals. The above situation is a special case of the dynamical phenomenon called phase locking. It is characteristic for one-parameter deformations in elliptic dynamics. See [77] for a study and a detailed discussion of this phase locking when $\gamma$ is a triangle.

Let now $\gamma$ be a convex polygon. Then the $C^{1}$ curve $G(\gamma, \ell)$ is a concatenation of arcs of ellipses with foci at the corners of $\gamma$. At the points of transition between these elliptic arcs, typically, only one of the two foci changes, causing a jump in the curvature. Thus, a typical billiard table, say $Y$, obtained by this construction, is strictly convex, piecewise analytic, but not $C^{2}$. The boundary $\partial Y$ contains a finite number of points where the curvature jumps. A. Hubacher studied billiard tables of this class [92]. 
She proved that there is an open neighborhood $\Omega \subset Y$ of $\partial Y$ such that any caustic in $Y$ belongs to the complement of $\Omega$ [92].

Recall that $\gamma$ is a caustic of $Y(\gamma, \ell)$ for any $\ell>|\gamma|$. There is an analogy between Hubacher's theorem and a result in [76 which says that billiard caustics stay away from the table's boundary if it contains points of very small curvature. This result is a quantitative version of Mather's theorem 114 that insures nonexistence of caustics if $\partial Y$ has points of zero curvature. Hubacher's theorem replaces them with jump points of the curvature. As opposed to [76], the work [92] does not estimate the size of the region $\Omega \subset Y$ free of caustics. It is plausible that for the typical table $Y(\gamma, \ell)$ the free of caustics region $\Omega=\Omega(\gamma, \ell)$ is the annulus between $\partial Y(\gamma, \ell)$ and $\gamma$.

There are polygons $\gamma$ such that for special values of the string length $\ell$ the boundary $\partial Y(\gamma, \ell)$ is a $C^{2}$ curve. Let $\gamma$ be the regular hexagon with the unit side length. Then $\partial Y(\gamma, 14)$ is a $C^{2}$ curve. The work of H. Fetter [43] studies the billiard on $Y(\gamma, 14)$. Fetter suggests that the billiard on $Y(\gamma, 14)$ is integrable, and thus $Y(\gamma, 14)$ is a counterexample to the Birkhoff conjecture. However, the evidence of integrability of $Y(\gamma, 14)$ presented in 43 is mostly numerical. The present author believes that the further investigation of the billiard on $Y(\gamma, 14)$ will confirm the Birkhoff conjecture.

The subject of 97 is a billiard version of the famous question of Marc Kac: "Can one hear the shape of a drum?" 96. As an application of their results, the authors prove a conditional version of the Birkhoff conjecture.

\subsection{The Ivrii conjecture and related material.}

Investigations of Problem 3i) (i.e., the Ivrii conjecture) and Problem 3ii) (i.e., the claims $I_{n}$ for $n>2$ ), as well as related questions, remain active. The work [8] develops a functional theoretic approach to study billiard caustics. As a byproduct, [8] contains yet another proof of Rychlik's theorem stating that the set of 3 -periodic billiard orbits has measure zero. The preprint 65. announced a solution of the Ivrii conjecture. Unfortunately, the work [65. contains a mistake; thus the conjecture remains open. The paper [51] announced a proof of the claim $I_{4}$ : The set of 4-periodic billiard orbits has measure zero. The work [51] states several propositions implying the claim, and explains the strategy of their proofs. The approach of [51] is based on a study of certain foliations, on one hand, and a very detailed analysis of singularities of certain mappings, on the other hand. Complete proofs should appear shortly. There is a curious connection between the Ivrii conjecture and the subject of invisibility [120].

It is natural to investigate the counterparts of the Ivrii conjecture for the billiard on (simply connected) surfaces of constant curvature. The billiard on $\mathbb{R}^{2}$ corresponds to the zero curvature, $\kappa=0$. Multiplying a constant curvature by a positive factor does not qualitatively change the geometry; thus, it suffices to consider the two cases $\kappa= \pm 1$. The surfaces in question are the hyperbolic plane, $\kappa=-1$, and the round unit sphere, $\kappa=1$. Let us denote them by $\mathbb{H}^{2}$ and $\mathbb{S}^{2}$ respectively. On $\mathbb{S}^{2}$ the immediate analog of the 
Ivrii conjecture fails. The paper [80] contains an example of a (not strictly) smooth, convex billiard table in $\mathbb{S}^{2}$ with an open set of periodic orbits. This observation shows the subtlety of the Ivrii conjecture for $\mathbb{R}^{2}$. The work 13 . contains a detailed study of 3-periodic orbits for billiard tables in $\mathbb{H}^{2}$ and $\mathbb{S}^{2}$. It shows, in particular, that the set of 3 -periodic billiard orbits on a Birkhoff billiard table in $\mathbb{H}^{2}$ has measure zero. This is the counterpart of Rychlik's theorem for the hyperbolic plane.

\subsection{Extensions of the material in $\S \mathbf{2}$.}

Nontrivial billiard properties can be roughly divided into three categories: i) Those that hold for all tables; ii) Those that hold for the typical table; iii) Those that hold for special billiard tables. Studies in category iii) can be described as follows: Let $\mathrm{P}$ be a property satisfied by a very particular billiard table, e. g., the round disc. Are there non-round tables that have property P? If the answer is "yes", then describe the billiard tables having property $\mathrm{P}$.

The following example illustrates the situation. Let $0<\alpha \leq \pi / 2$ be an angle. Let $Y \subset \mathbb{R}^{2}$ be a Birkhoff billiard table. We say that the table $Y$ has property $P_{\alpha}$ if every chord in $Y$ that makes angle $\alpha$ with $\partial Y$ at one end also makes angle $\alpha$ with $\partial Y$ at the other end. The round table has property $P_{\alpha}$ for any $\alpha$. A billiard table with property $P_{\alpha}$ has a very special caustic $\Gamma_{\alpha}$; we will say that $\Gamma_{\alpha}$ is a constant angle caustic. Let $\rho(\theta), 0 \leq \theta \leq 2 \pi$, be the radius of curvature for $\partial Y$. Tables with the caustic $\Gamma_{\pi / 2}$ are well known to geometers: Their boundaries are the curves of constant width [9, 52]. A curve $\partial Y$ has constant width if an only if its radius of curvature satisfies the identity

$$
\rho(\theta)+\rho(\theta+\pi)=\text { const. }
$$

In particular, there are non-round infinitely smooth, and even real analytic billiard tables in this class.

For $0<\alpha<\pi / 2$ let $\mathcal{P}_{\alpha}$ be the class of non-round tables with the property $P_{\alpha}$. The author has investigated the class $\mathcal{P}_{\alpha}$ about 20 years ago and reported the results at the 1993 Pennsylvania State University Workshop on Dynamics [59]. The main results in [59] are as follows: The class $\mathcal{P}_{\alpha}$ is nonempty if and only if $\alpha$ satisfies

$$
\tan (n \alpha)=n \tan \alpha
$$

for some $n>1$. The set $A_{n} \subset(0, \pi / 2)$ of solutions of equation (6) is finite and nonempty for $n \geq 4$. For every $\alpha \in A_{n}$ there is an analytic family of (nonround) distinct, convex, real analytic tables $Y_{\alpha}(s) \in \mathcal{P}_{\alpha}: 0<s<1$. As $s \rightarrow 0$, the tables $Y_{\alpha}(s)$ converge to the unit disc. The limit $Y_{\alpha}(1)$ of $Y_{\alpha}(s)$, as $s \rightarrow 1$, also exists, but has corners.

It turns out that planar regions satisfying property $P_{\alpha}$ for some angle $\alpha$ are of interest in the mathematical fluid mechanics. Besides the concept of Archimedean floating 10 there is a concept of capillary floating in neutral

\footnotetext{
${ }^{10}$ In fact, this concept goes back to Aristotle.
} 
equilibrium at a particular contact angle. This concept goes back to Thomas Young [154] and was further developed and investigated by R. Finn [44]. If $Y \in \mathcal{P}_{\alpha}$, then the infinite cylinder $C=Y \times \mathbb{R}$ floats in neutral equilibrium at the contact angle $\pi-\alpha$ at every orientation. The work 64 is a detailed exposition of the results in 59] aimed, in particular, at the mathematical fluid mechanics readership. See [140, 31] for related investigations and 69] for additional comments.

\subsection{Comments and updates for the material in $\S 4$.}

Although Problem 10 remains open, recent publications [127, 128, 91] provided substantial evidence toward the positive answer, i.e., that every polygon does have a periodic orbit. These papers investigate periodic billiard orbits in obtuse triangles. Let $\Delta(\alpha, \beta, \gamma)$ be the triangle with the angles $\alpha \leq \beta \leq \gamma$. In [127, 128] R. Schwartz proves that if $\gamma$ is less than or equal 100 degrees, then $\Delta(\alpha, \beta, \gamma)$ has a periodic orbit. In 91] Hooper and Schwartz prove that if the angles $\alpha$ and $\beta$ are sufficiently close, then $\Delta(\alpha, \beta, \gamma)$ has a periodic orbit. If $\alpha=\beta$, then $\Delta$ is an isosceles triangle. It is well known and elementary that isosceles triangles have periodic orbits. The main theorem in [91] says that any triangle which is sufficiently close to an isosceles one, has periodic orbit. Note that in the Hooper-Schwartz theorem the angles $\alpha$ and $\beta$ can be arbitrarily small, thus $\gamma$ can be arbitrarily close to 180 degrees.

Let $\Delta$ be a triangle, and let $a, b, c$ be the sides of $\Delta$. The works [127, 128, 91 build on the approach of [47, where periodic billiard orbits were coded by words on the alphabet $\{a, b, c\}$. The paper [47] studied relationships between periodic orbits and the associated words. A periodic billiard orbit is stable if it persists under all sufficiently small deformations of $\Delta$. By [47, the stability of an orbit is equivalent to a combinatorial property of the associated word. For the sake of brevity, I will simply say that the words on the alphabet $\{a, b, c\}$ satisfying this property are stable. Let $\mathcal{T} \subset \mathbb{R}^{2}$ be the moduli space of triangles. Slightly simplifying the situation, we assume that $\mathcal{T}=\left\{(x, y) \in \mathbb{R}^{2}: 0 \leq x, y \leq 1\right\}$. The subsets of obtuse (resp. isosceles) triangles are given by $x+y<1$ (resp. $x=y$ ). Let $W_{k}$ be the set of stable words of length $k$, and let $W=\cup_{k} W_{k}$. For $w \in W$ let $\mathcal{T}_{w} \subset \mathcal{T}$ be the open set of triangles having a periodic orbit with the code $w$. These are the tiles in the terminology of [91].

The approach of Hooper and Schwartz is to exhibit a sufficient set $W_{\text {suff }} \subset$ $W$ so that the tiles $\left\{\mathcal{T}_{w}: w \in W_{\text {suff }}\right\}$ cover the targeted part of $\mathcal{T}$. It goes without saying that this idea cannot be implemented without substantial computer power. The computer program "MacBilliards" created by Hooper and Schwartz does the job. Besides providing us with ample evidence towards the conjecture that every polygon has periodic billiard orbits, the works 127, 128, 91 have established several facts that show just how intricate the matter is. In some cases, every finite set is insufficient; then [91] sufficient infinite sets. 
Unfortunately, the survey 62 has omitted the subject of complexity of billiard orbits in polygons, which is very close to Problem 11 . We will briefly discuss it below. Let $P$ be a polygon, and let $\mathcal{A}=\{a, b, c, \ldots\}$ be the set of its sides. Following a finite billiard orbit $\gamma$ and recording the sides that it encounters, we obtain a word, $w(\gamma)$, on the alphabet $\mathcal{A}$. We say that $w(\gamma)$ is the code of $\gamma$; the number of letters in $w(\gamma)$ is the combinatorial length of $\gamma$. Let $W_{n}(P)$ be the set of codes of all billiard orbits with combinatorial length $n$. The function $F(n)=\left|W_{n}(P)\right|$ is the full complexity of the billiard on $P$. Imposing various restrictions on the billiard orbits, we obtain conditional or partial complexities $F_{*}(n)$. For instance, the function $f_{P}(n)$ in Problem 11 is the periodic complexity for the billiard on $P$. Thus, Problem 11 is a special case of the following open question.

Problem 14. Find nontrivial, explicit bounds on partial billiard complexities for the general polygon 11

By [99, 70, 71, the full complexity of the billiard in any polygon is subexponential. This means that for $n$ sufficiently large $F(n)<e^{a n}$ for any $a>0$. Note that this result provides no subexponential bound on $F(n)$. Very few nontrivial bounds on billiard complexities are known. Let $f_{P}(n)$ be the number of codes for periodic orbits in $P$ of length at most $n$. For any $k \in \mathbb{N}$ Hooper constructed open sets $\mathcal{X}_{k}$ in the moduli space of polygons such that for $P \in \mathcal{X}_{k}$ the function $f_{P}(n)$ grows faster than $n \log ^{k} n$ [88]. Let $g_{P}(n)$ be the number of generalized diagonals in $P$ of length at most $n$. Let $\mathcal{T}_{3}$ be the moduli space of triangles, endowed with the Lebesgue measure. Scheglov [125] showed that for almost every $P \in \mathcal{T}_{3}$ and any $\varepsilon>0$ the inequality $g_{p}(n)<$ const $\exp \left(n^{\sqrt{3}-1+\varepsilon}\right)$ holds. Note that Scheglov's result yields explicit subexponential upper bounds on the full and the periodic complexities for almost every triangle.

To make Problem 14 more concrete, we state below a widely accepted conjecture.

Conjecture 1. There is $d \geq 3$ such that the full billiard complexity for any polygon has a cubic lower bound and a degree $d$ upper bound.

The claim is established only for rational polygons, with $d=3$. This is a consequence of the results of Masur [110, 111] about saddle connections on translation surfaces. We will now briefly discuss recent results on partial complexities that provide support for it 78 . Let $P$ be an arbitrary polygon. Let $0 \leq \theta<2 \pi$ be a direction, and let $z \in P$ be a point. Coding those billiard orbits that start off in direction $\theta$ (resp. from the point $z$ ) and counting the number of words of length less than or equal to $n$, we obtain the directional complexity $F_{\theta}(n)$ (resp. position complexity $F_{z}(n)$ ) for the billiard on $P$. It was known that the directional complexity grows polynomially, i.e., there

\footnotetext{
${ }^{11}$ The designation general polygon may be replaced by irrational polygon.
} 
is $d>0$ depending only on $P$ such that $F_{\theta}(n)=O\left(n^{d}\right)$ for all directions $\theta$ [82. By [78, for any $P$ and any $\varepsilon>0$ for almost all directions $\theta$, we have $F_{\theta}(n)=O\left(n^{1+\varepsilon}\right)$. Another result in [78 says that for any $P$ and any $\varepsilon>0$, for almost all points $z \in P$ we have $F_{z}(n)=O\left(n^{2+\varepsilon}\right) 12$ The proofs are based on the relationships between the average complexity and individual complexities. The concept of a piecewise convex polygon exchange introduced in 81 yields a new approach to the billiard complexities. This approach works for the polygonal billiard on surfaces of arbitrary constant curvature.

\subsection{Ramifications and extensions of the polygonal billiard.}

In this section we briefly report on several generalizations of the subject

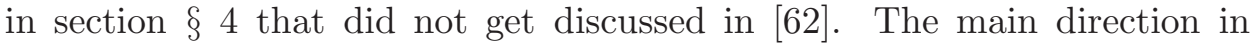
these developments is to replace the usual polygons by noncompact or infinite polygons. The work in this direction started already at the end of the last century [33, 34, and flourished after the turn of the century. The noncompact polygons in [33, 34] are semi-infinite stairways. A stairway in these works is a noncompact polygon, say $P$, with infinitely many vertical and horizontal sides, and of finite area. In particular, $P$ is a rational noncompact polygon, and we have the obvious family of directional billiard flows $b_{\theta}^{t}, 0 \leq \theta \leq \pi / 2$, on $P$. In addition to studying the ergodicity of these flows, the papers [33, 34] investigate escaping orbits in $P$, a new phenomenon caused by the noncompactness of $P$.

There are many kinds of noncompact polygons, both rational and irrational, bounded or unbounded, with finite or infinite area. More generally, there are noncompact polygonal surfaces [67, and in particular, noncompact translation surfaces [89, 67. In this brave new world, there are infinite (as opposed to semi-infinite) stairways [90, infinite coverings of compact polygonal surfaces [67, 46, 30], and even fractals, e.g., the Koch snowflake [108. Besides being of interest on its own, the billiard on noncompact polygons comes up in physics and engineering [54, 56, 58, 32, 6].

All of the questions concerning the dynamics and geometry for compact polygons, translation surfaces, etc, have obvious counterparts in the noncompact world 13 The answers to these questions are sometimes unexpected [46, 30, 142, not at all analogous to the answers in the compact world. Besides, there are problems in the noncompact world that do not arise in the classical setting. One of them is the conservativity of billiard dynamics. In the classical setting the conservativity is ensured by the Poincaré recurrence theorem. We conclude this necessarily incomplete survey of noncompact polygonal billiard with a brief discussion of the billiard for a classical family of noncompact, doubly periodic polygons.

\footnotetext{
12 This establishes, in particular, the unpublished results of Boshernitzan [16].

${ }^{13}$ Except for the billiard on a fractal table where these counterparts are not obvious 108.
} 
Let $0<a, b<1$, and let $R(a, b)$ be the $a \times b$ rectangle. Denote by $R_{(0,0)}$ the upright rectangle $R(a, b)$ centered at $(1 / 2,1 / 2)$. For $(m, n) \in \mathbb{Z}^{2}$ set $R_{(m, n)}=R_{(0,0)}+(m, n)$. The region $\tilde{P}(a, b)=\mathbb{R}^{2} \backslash\left(\cup_{(m, n) \in \mathbb{Z}^{2}} R_{(m, n)}\right)$ is a noncompact rational polygon of infinite area. We will refer to it as the rectangular Lorenz gas. The randomized version of $\tilde{P}(a, b)$ is the famous wind-tree model of statistical physics [38]. For $0 \leq \theta<\pi / 2$ denote by $b_{\theta}^{t}$ the directional billiard flows on $\tilde{P}(a, b)$. The work 30] works out the ergodic decomposition of $b_{\theta}^{t}$ on $\tilde{P}(a, b)$ for particular directions $\theta$ and sufficiently small $a$ and $b$, provided that $a / b$ be irrational. The directions in question are $\theta=\arctan (q / p)$ corresponding to $(p, q) \in \mathbb{N}^{2}$ such that the flow line of $b_{\theta}^{t}$ emanating from a corner of $R_{(0,0)}$ reaches the homologous corner of $R_{(p, q)}$ bypassing the obstacles. Although these directions form a finite set, it is asymptotically dense as $a, b$ go to zero. The dissipative component of $b_{\theta}^{t}$ is spanned by the straight lines 14 avoiding the obstacles. The conservative part of $b_{\theta}^{t}$ decomposes as a direct sum of $2 p q$ identical ergodic flows. The decomposition is as follows. There is a certain subgroup $H_{(p, q)} \subset \mathbb{Z}^{2}$ of index $2 p q$. Let $S_{1}, \ldots, S_{2 p q} \subset \mathbb{Z}^{2}$ be its cosets. The ergodic component of $b_{\theta}^{t}$ corresponding to the coset $S_{i}, 1 \leq i \leq 2 p q$, is spanned by the billiard orbits that encounter the obstacles $R_{(m, n)}$, where $(m, n) \in S_{i}$. Thus, the ergodic decomposition of the flow in the $(p, q)$ direction is induced by a natural partition of the set of obstacles in the configuration space. For instance, the two ergodic components of the flow in the direction $\pi / 4$ correspond to the billiard orbits on $\tilde{P}(a, b)$ that encounter the obstacles $R_{(m, n)}$ with even and odd $m+n$ respectively.

These results follow from the ergodicity of certain $\mathbb{Z}^{2}$-valued cocycles over irrational rotations [30] established by the classical methods of ergodic theory for infinite invariant measures [1, 29, 126]. The explicit ergodic decomposition of the (conservative part of) the directional flows yields nontrivial consequences for the recurrence and the spatial distribution of typical billiard orbits [30]. The recurrence in the direction $\pi / 4$ has been discussed in the physics literature [84]. Judging by the results in [30, 46] etc, the noncompact polygonal billiard may yield further surprises.

\subsection{Security for billiard tables and related questions.}

This subject arose quite recently. It has to do with the geometry of billiard orbits as curves on the configuration space. Let $\Omega \subset \mathbb{R}^{2}$ be any billiard table. For any pair $x, y \in \Omega$ (in particular for pairs $x, x$ ) let $\Gamma(x, y)$ be the set of billiard orbits in $\Omega$ that connect $x$ and $y$. We say that the pair $x, y$ is secure if there exists a finite set $\left\{z_{1}, \ldots, z_{n}\right\} \subset \Omega \backslash\{x, y\}$ such that every $\gamma \in \Gamma(x, y)$ passes through some $z_{i}$. We say that $z_{1}, \ldots, z_{n}$ are blocking points for $x, y$. We call $\Omega$ secure if every pair of points is secure. Thus, to show that $\Omega$ is insecure means to find a pair $x, y$ that cannot be blocked with a finite set of blocking points. The questions that arise are: i) What

\footnotetext{
${ }^{14}$ With the slopes $\pm q / p$.
} 
tables $\Omega$ are secure?; ii) If $\Omega$ is insecure, how insecure is it? For instance, is it true that every pair $x, y \in \Omega$ is insecure, that almost all pairs $x, y \in \Omega$ are insecure, etc.

The subject, in disguise of problems about bodyguards came up in the Mathematical Olympiad literature. The recent interest in security got triggered by [87]. The authors, who were then students at Cambridge University, studied the security of polygons. The main claim in [87] is that every rational polygon is secure. Regular $n$-gons provide a counterexample to the claim: A regular $n$-gon is secure if and only if $n=3,4,6$ [63]. See 64] for related results. Although the statement is elementary, the proof is not. The claim follows from a study of security in translation surfaces, and is based on [55, 143, 145, 73, 74, 72. However, the general study of security for polygons has just begun.

Problem 15. To characterize secure polygons. In particular, establish a criterion of security for rational polygons.

Triangles with the angles of 30,60,90 and 45,45,90 degrees, as well as the equilateral triangle and the rectangles are the only polygons whose translation surfaces are flat tori, and hence their billiard flows are integrable [55]. Following [55, we will call them integrable polygons. A polygon $P$ is almost integrable if it is tiled under reflections by one of the integrable polygons [75]. By [63], every almost integrable polygon is secure.

Conjecture 2. A polygon is secure if and only if it is almost integrable.

Almost integrable polygons are certainly rational. Conjecture 2 would imply, in particular, that every irrational polygon is insecure. At present, the problem of insecurity for general polygons seems hopeless. However, the security framework makes sense for arbitrary billiard tables, and more generally, for arbitrary riemannian manifolds (with boundary, corners, and singularities, in general). Security of riemannian manifolds is related to the growth of the number of connecting geodesics, and hence to their topological entropy [21, 66]. Not much is known about the security in non-polygonal billiard tables $\Omega$. Let $\Omega$ be a Birkhoff billiard table. Approximating $\partial \Omega$ locally by the arcs of its osculating circles, it is intuitively clear that pairs of sufficiently close points $x, y \in \partial \Omega$ are insecure; the work [141] confirms it.

Much more is known about the security of compact, smooth Riemannian manifolds. Flat manifolds are secure [63, 21]. For a flat torus, the proof is elementary [63]. For general flat manifolds, this follows from the Bieberbach theorem [21].

Conjecture 3. A compact, smooth riemannian manifolds is secure if and only if it is flat. 
Conjecture 3 has been established for various classes of compact Riemannian manifolds [21, 79, 7]. In particular, it holds for all compact surfaces of genus greater than zero [7]. Thus, among surfaces, it remains to prove it for arbitrary smooth Riemannian metrics on the two-sphere. The work [49] gives examples of totally insecure real analytic metrics on the twosphere; 48] shows that higher dimensional compact Riemannian manifolds are generically insecure.

Acknowledgements. The author gratefully acknowledges discussions with many mathematicians concerning this survey and the comments of anonymous referees. The work was partially supported by the MNiSzW Grant N N201 384834.

\section{REFERENCES}

[1] J. Aaronson, An Introduction to Infinite Ergodic Theory, A. M. S., Providence, RI, 1997.

[2] E. Amiran, Caustics and evolutes for convex planar domains J. Differential Geom. 28 (1988), 345 - 357.

[3] S. Angenent, A remark on the topological entropy and invariant circles of an area preserving twist map, Proceedings IMA, Vol. 44 (1992), 1 - 6.

[4] R. Artuso, G. Casati, I. Guarneri, Numerical study on ergodic properties of triangular billiards, Phys. Rev. E 55 (1997), 6384 - 6390.

[5] A. Avila, Chaoticity of the Teichmller flow, in "Homogeneous flows, moduli spaces and arithmetic", 321 - 338, Clay Math. Proc., 10, Amer. Math. Soc., Providence, RI, 2010.

[6] P. Bachurin, K. Khanin, J. Marklof, A. Plakhov, Perfect retroreflectors and billiard dynamics, J. Mod. Dyn. 5 (2011), 33 - 48.

[7] V. Bangert and E. Gutkin, Insecurity for compact surfaces of positive genus, Geom. Dedicata 146 (2010), 165 - 191.

[8] Yu. Baryshnikov, V. Zharnitsky, Sub-Riemannian geometry and periodic orbits in classical billiards, Math. Res. Lett. 13 (2006), 587 - 598.

[9] M. Berger, Geometry revealed. A Jacob's ladder to modern higher geometry, Springer, Heidelberg, 2010.

[10] M. Bialy, Convex billiards and a theorem by E. Hopf, Math. Zeitschr. 214 (1993), $147-154$.

[11] M. Bialy, Hopf rigidity for convex billiards on the hemisphere and hyperbolic plane, preprint arXiv:1205.3873 (2012).

[12] G.D. Birkhoff, Dynamical Systems, A.M.S., Providence 1927; $<$ http://www.ams.org/bookstore/collseries $>$.

[13] V. Blumen, K.Y. Kim, J. Nance, V. Zharnitsky, Three-period orbits in billiards on the surfaces of constant curvature, preprint arXiv:1108.0987v2 (2011).

[14] S.V. Bolotin, Integrable Birkhoff billiards, Vestnik Moskov. Univ. Ser. I Mat. Mekh. (1990), 33 - 36. (in Russian)

[15] M. Boshernitzan, A condition for minimal interval exchange maps to be uniquely ergodic, Duke Math. J. 52 (1985), 733 - 752.

[16] M. Boshernitzan, Unpublished Manuscript.

[17] L. Bunimovich, On the ergodic properties of certain billiards, Func. Analys. \& Appl. 8 (1974), $254-255$.

[18] L. Bunimovich, Billiards and other hyperbolic systems, pp. 192 - 233 in Encyclopedia of Mathematical Sciences vol. 100, Springer-Verlag, New York 2000. 
[19] L. Bunimovich, Ya. Sinai, N. Chernov, Markov partitions for two-dimensional hyperbolic billiards, Russ. Math. Surv. 45 (1990), 105 - 152.

[20] L. Bunimovich, Ya. Sinai, N. Chernov, Statistical properties of two-dimensional hyperbolic billiards, Russ. Math. Surv. 46 (1991), 47 - 106.

[21] K. Burns and E. Gutkin, Growth of the number of geodesics between points and insecurity for Riemannian manifolds, Discrete Contin. Dyn. Syst. 21 (2008), 403 413.

[22] G. Casati and T. Prosen, Mixing property of triangular billiards, Phys. Rev. Lett. 83 (1999), 4729 - 4732.

[23] N. Chernov, Topological entropy and periodic points of two-dimensional hyperbolic billiards, Funct. Anal. Appl. 25 (1991), 39 - 45.

[24] N. Chernov, Entropy, Lyapunov exponents and mean-free path for billiards, J. Stat. Phys. 88 (1997), 1 - 29.

[25] N. Chernov, Decay of correlations in dispersing billiards, J. Stat. Phys. 94 (1999), $513-556$.

[26] N. Chernov, R. Markarian, Chaotic billiards, American Mathematical Society, Providence, RI, 2006.

[27] Y. Cheung, Hausdorff dimension of the set of nonergodic directions, with an appendix by M. Boshernitzan, Ann. Math. 158 (2003), 661 - 678.

[28] B. Cipra, R. Hanson, E. Kolan, Periodic trajectories in right-triangle billiards, Phys. Rev. E 52 (1995), 2066 - 2071.

[29] J.-P. Conze, Recurrence, ergodicity and invariant measures for cocycles over a rotation, Contemporary Mathematics 485, A.M.S., Providence, RI, (2009), 45 - 70.

[30] J.-P. Conze and E. Gutkin, On recurrence and ergodicity for geodesic flows on noncompact periodic polygonal surfaces, Erg. Theor. \& Dyn. Syst. 32 (2012), 491 - 515.

[31] V. Cyr, A number theoretic question arising in the geometry of plane curves and in billiard dynamics, Proc. A.M.S. 140 (2012), 3035 - 3040.

[32] M. Daniel and E. Gutkin, The dynamics of a generalized Heisenberg ferromagnetic spin chain, Chaos: Interdiscipl. Journ. Nonlin. Science 5 (1995), 439 - 443.

[33] M. Degli Esposti, G. Del Magno, M. Lenci, An infinite step billiard, Nonlinearity 11 (1998), $991-1013$.

[34] M. Degli Esposti, G. Del Magno, M. Lenci, Escape orbits and ergodicity in infinite step billiards, Nonlinearity 13 (2000), 1275 - 1292.

[35] R. de la Llave, A tutorial on KAM theory, 175 - 292, Proc. Sympos. Pure Math. 69, Amer. Math. Soc., Providence, RI, 2001; $<$ ftp://ftp.ma.utexas.edu/pub/papers/llave/tutorial.pdf $>$.

[36] L. DeMarco, The conformal geometry of billiards, Bull. Amer. Math. Soc. 48 (2011), $33-52$.

[37] R. Douady, Applications du théorème des tores invariants, Univ. Paris 7, Thèse 3-eme cycle, 1982.

[38] P. Ehrenfest and T. Ehrenfest, Encyclopedia article (1912); english translation in "The conceptual foundations of the statistical approach in mechanics", Cornell University Press, Ithaca, N.Y., 1959.

[39] A. Eskin, H. Masur, Asymptotic formulas on flat surfaces, Erg. Theor. \& Dyn. Sys. 21 (2001), $443-478$.

[40] A. Eskin, H. Masur, M. Schmoll, Billiards in rectangles with barriers, Duke Math. J. 118 (2003), 427 - 463.

[41] A. Eskin, H. Masur, A. Zorich, Moduli spaces of abelian differentials: The principal boundary, counting problems and the Siegel - Veech constants, Publ. Math. Inst. Hautes Études Sci. 97 (2003), 61 - 179.

[42] A. Eskin, A. Okunkov, Asymptotics of numbers of branched coverings of a torus and volumes of moduli spaces of holomorphic differentials, Invent. Math. 145 (2001), 59 - 103. 
[43] H.L. Fetter, Numerical exploration of a hexagonal string billiard, Physica D 241 (2012), $830-846$.

[44] R. Finn, Floating bodies subject to capillary attractions, J. Math. Fluid Mech. 11 (2009), $443-458$.

[45] R.H. Fox and R.B. Kershner, Concerning the transitive properties of geodesics on a rational polyhedron, Duke Math. J. 2 (1936), 147 - 150.

[46] K. Fraczek, C. Ulcigrai, Non-ergodic Z-periodic billiards and infinite translation surfaces, arXiv:1109.4584 (2011).

[47] G. Galperin, A. Stepin, Ya. Vorobets, Periodic orbits in polygons: generating mechanisms, Russ. Math. Surv. 47 (1992), 5 - 80.

[48] M. Gerber and Wah-Kwan Ku, A dense G-delta set of Riemannian metrics without the finite blocking property, Math. Res. Letters 18 (2011), 389 - 404.

[49] M. Gerber and Lihuei Liu, Real analytic metrics on $S^{2}$ with total absence of finite blocking, preprint arXiv:1109.1336 (2011).

[50] S.L. Glashow and L. Mittag, Three rods on a ring and the triangular billiard, J. Stat. Phys. 87 (1997), 937 - 941.

[51] A.A. Glutsyuk and Yu.G. Kudryashov, On quadrilateral orbits in planar billiards, Dokl. Math. 83 (2011), 371 - 373.

[52] P.M. Gruber, Convex and discrete geometry, Springer, Berlin, 2007.

[53] B. Gutkin, U. Smilansky, E. Gutkin, Hyperbolic billiards on surfaces of constant curvature, Comm. Math. Phys. 208 (1999), 65 - 90.

[54] E. Gutkin and M. Kac, Propagation of chaos and the Burgers equation, SIAM Journ. Appl. Math. 43 (1983), 971 - 980.

[55] E. Gutkin, Billiard flows on almost integrable polyhedral surfaces, Erg. Theor. \& Dyn. Sys. 4 (1984), 560 - 584.

[56] E. Gutkin, Asymptotics of trajectories for cone potentials, Phys. D 17 (1985), 235 $-242$.

[57] E. Gutkin, Billiards in polygons, Physica D 19 (1986), 311 - 333.

[58] E. Gutkin, Continuity of scattering data for particles on the line with directed repulsive interactions, Journ. Math. Phys. 28 (1987), 351 - 359.

[59] E. Gutkin, Billiard tables of constant width and the dynamical characterizations of the circle, Workshop on Dynamics and Related Topics, PennState U. (1993), 21 24.

[60] E. Gutkin, Billiards in polygons: Survey of recent results, J. Stat. Phys. 83 (1996), $7-26$.

[61] E. Gutkin, Two applications of calculus to triangular billiards, Amer. Math. Monthly 104 (1997), 618 - 623.

[62] E. Gutkin, Billiard dynamics: a survey with the emphasis on open problems, Regul. \& Chaot. Dyn. 8 (2003), 1 - 13.

[63] E. Gutkin, Blocking of billiard orbits and security for polygons and flat surfaces, GAFA: Geom. \& Funct. Anal. 15 (2005), 83 - 105.

[64] E. Gutkin, Insecure configurations in lattice translation surfaces, with applications to polygonal billiards, Discr. Contin. Dyn. Syst. 16 (2006), 367 - 382.

[65] E. Gutkin, A few remarks on periodic orbits for planar billiard tables, preprint arXiv:math/0612039 (2006).

[66] E. Gutkin, Topological entropy and blocking cost for geodesics in Riemannian manifolds, Geom. Dedicata 138 (2009), 13 - 23.

[67] E. Gutkin, Geometry, topology and dynamics of geodesic flows on noncompact polygonal surfaces, Reg. \& Chaot. Dyn. 15 (2010), 482 - 503.

[68] E. Gutkin, Capillary floating and the billiard ball problem, J. Math. Fluid Mech. 14 (2012), $363-382$.

[69] E. Gutkin, Addendum to: Capillary floating and the billiard ball problem, preprint (2012). 
[70] E. Gutkin and N. Haydn, Topological entropy of generalized polygon exchanges, Bull. Amer. Math. Soc. 32 (1995), 50 - 56.

[71] E. Gutkin and N. Haydn, Topological entropy of polygon exchange transformations and polygonal billiards, Erg. Theor. \& Dyn. Sys. 17 (1997), 849 - 867.

[72] E. Gutkin, P. Hubert, T. Schmidt, Affine diffeomorphisms of translation surfaces: periodic points, Fuchsian groups, and arithmeticity, Ann. Sci. Ecole Norm. Sup. 36 (2003), $847-866$.

[73] E. Gutkin and C. Judge, The geometry and arithmetic of translation surfaces with applications to polygonal billiards, Math. Res. Lett. 3 (1996), 391 - 403.

[74] E. Gutkin and C. Judge, Affine mappings of translation surfaces: geometry and arithmetic, Duke Math. J. 103 (2000), 191 - 213.

[75] E. Gutkin and A. Katok, Weakly mixing billiards, Holomorphic dynamics (Mexico, 1986), 163 - 176, Lecture Notes Math. 1345, Springer, Berlin, 1988.

[76] E. Gutkin and A. Katok, Caustics for inner and outer billiards, Comm. Math. Phys. 173 (1995), $101-133$.

[77] E. Gutkin and O. Knill, Billiards that share a triangular caustic, 199 - 213, Adv. Ser. Nonlinear Dynam. 8, World Sci. Publ., River Edge, NJ, 1996.

[78] E. Gutkin and M. Rams, Growth rates for geometric complexities and counting functions in polygonal billiards, Erg. Theor. \& Dyn. Sys. 29 (2009), 1163 - 1183.

[79] E. Gutkin and V. Schroeder, Connecting geodesics and security of configurations in compact locally symmetric spaces, Geom. Dedicata 118 (2006), 185 - 208.

[80] E. Gutkin and S. Tabachnikov, Billiards in Finsler and Minkowski geometries, J. Geom. \& Phys. 40 (2001/2), 277 - 301.

[81] E. Gutkin and S. Tabachnikov, Complexity of piecewise convex transformations in two dimensions, with applications to polygonal billiards on surfaces of constant curvature, Mosc. Math. J. 6 (2006), 673 - 701.

[82] E. Gutkin and S. Troubetzkoy, Directional flows and strong recurrence for polygonal billiards, Pittman Res. Notes in Math. $\mathrm{N}^{\circ} \mathbf{3 6 2}$ (1997), 21 - 45.

[83] L. Halbeisen and N. Hungerbühler, On periodic billiard trajectories in obtuse triangles, SIAM Review 42 (2000), 657 - 670.

[84] J. Hardy and J. Weber, Diffusion in a periodic wind-tree model, J. Math. Phys. 21 (1980), 1802 - 1808.

[85] G.H. Hardy and E.M. Wright, An introduction to the theory of numbers, Oxford U. Press, London 1938.

[86] B. Hasselblatt and A. Katok (editors), Handbook of Dynamical Systems, Volume 1A, Elsevier, Amsterdam 2002.

[87] P. Hiemer, V. Snurnikov, Polygonal billiards with small obstacles, J. Stat. Phys. 90 (1998), $453-466$.

[88] W.P. Hooper, Lower bounds on growth rates of periodic billiard trajectories in some irrational polygons, J. Mod. Dyn. 1 (2007), 649 - 663.

[89] W.P. Hooper, Dynamics on an infinite surface with the lattice property, preprint arXiv:0802.0189 (2000).

[90] W.P. Hooper, P. Hubert, B. Weiss, Dynamics on the infinite staircase, preprint (2010).

[91] W.P. Hooper, R.E. Schwartz, Billiards in nearly isosceles triangles, J. Mod. Dyn. 3 (2009), 159 - 231.

[92] A. Hubacher, Instability of the boundary in the billiard ball problem, Comm. Math. Phys. 108 (1987), 483 - 488.

[93] Y. Imayoshi, M. Taniguchi, An introduction to Teichmüller spaces, Springer-Verlag, Tokyo, 1992.

[94] N. Innami, Convex curves whose points are vertices of billiard triangles, Kodai Math. J. 11 (1988), 17 - 24. 
[95] V. Ivrii, The second term of the spectral asymptotic expansion for the LaplaceBeltrami operator on manifolds with a boundary, Func. Anal. \& Appl. 14 (1980), 98 - 106.

[96] M. Kac, Can one hear the shape of a drum?, Amer. Math. Monthly 73 (1966), 1 23.

[97] V. Kaloshin and A. Sorrentino, On conjugacy of convex billiards, preprint arXiv:1203.1274 (2012).

[98] A. Katok, Lyapunov exponents, entropy and periodic orbits for diffeomorphisms, Inst. Hautes Etudes Sci. Publ. Math. 51 (1980), 137 - 173.

[99] A. Katok, The growth rate for the number of singular and periodic orbits for a polygonal billiard, Comm. Math. Phys. 111 (1987), 151 - 160.

[100] A. Katok, The billiard table as a mathematician's playground, (in Russian), Publications of the Moscow Center for Continuing Mathematical Education, 2002.

[101] A. Katok (With the collaboration of Keith Burns), Infinitesimal Lyapunov functions, invariant cone families and stochastic properties of smooth dynamical systems, Erg. Theor. \& Dyn. Sys. 14 (1994), 757 - 785.

[102] A. Katok and B. Hasselblatt, Introduction to the modern theory of dynamical systems, CUP, Cambridge 1995.

[103] A. Katok, J.-M. Strelcyn, Invariant manifolds, entropy and billiards; smooth maps with singularities, LNM N ${ }^{o} \mathbf{1 2 2 2}$, Springer-Verlag, Berlin 1986.

[104] A. Katok, A. Zemlyakov, Topological transitivity of billiards in polygons, Math. Notes 18 (1975), $760-764$.

[105] R. Kenyon and J. Smillie, Billiards on rational-angled triangles, Comment. Math. Helv. 75 (2000), 65-108.

[106] S. Kerckhoff, H. Masur, J. Smillie, Ergodicity of billiard flows and quadratic differentials, Ann. Math. 124 (1986), 293 - 311.

[107] V. Kozlov and D. Treshchev, Billiards, A. M. S., Providence 1991.

[108] M.L. Lapidus, R.G. Niemeyer Sequences of compatible periodic hybrid orbits of prefractal Koch snowflake billiards, preprint arXiv:1204.3133 (2012).

[109] V. Lazutkin, The existemce of caustics for the billiard problem in a convex domain, Math. USSR Izv. 7 (1973), 185 - 214.

[110] H. Masur, Lower bounds for the number of saddle connection and closed trajectories of a quadratic differential, in "Holomorphic Functions and Moduli", vol. 2, SpringerVerlag, New York 1988.

[111] H. Masur, The growth rate of trajectories of a quadratic differential, Erg. Theor. \& Dyn. Sys. 10 (1990), 151-176.

[112] H. Masur and J. Smillie, Hausdorff dimension of sets of nonergodic measured foliations, Ann. Math. 134 (1991), 455 - 543.

[113] H. Masur, S. Tabachnikov, Rational billiards and flat structures, pp. 1015 - 1089 in Handbook of Dynamical Systems, Elsevier, Amsterdam 2002.

[114] J. Mather, Glancing billiards, Erg. Theor. \& Dyn. Sys. 2 (1982), 397 - 403.

[115] J. Moser, On invariant curves of area-preserving mappings of an annulus, Nachr. Akad. Wiss. Göttingen Math.-Phys. Kl. II (1962), 1 - 20.

[116] J. Moser, Remark on the paper: "On invariant curves of area-preserving mappings of an annulus", Nachr. Akad. Wiss. Göttingen Math.-Phys. Kl. II (1962), 1 - 20, Regul. Chaotic Dyn. 6 (2001), 337 - 338.

[117] V.I. Oseledets, A multiplicative ergodic theorem. Lyapunov characteristic numbers for dynamical systems, Trans. Moscow Math. Soc. 19 (1968), 197 - 231.

[118] J.C. Oxtoby, Measure and category, Springer-Verlag, New York 1996.

[119] V. Petkov and L. Stojanov, On the number of periodic reflecting rays in generic domains, Erg. Theor. \& Dyn. Sys. 8 (1988), 81 - 91.

[120] A. Plakhov, V. Roshchina, Invisibility in billiards, Nonlinearity 24 (2011), 847 - 854. 
[121] H. Poritsky, The billiard ball problem on a table with a convex boundary - An illustrative dynamical problem, Ann. Math. 51 (1950), 446 - 470.

[122] J.-Ch. Puchta, On triangular billiards, Comment. Mathem. Helv. 76 (2001), 501 505.

[123] M. Rychlik, Periodic points of the billiard ball map in a convex domain, J. Diff. Geom. 30 (1989), 191 - 205.

[124] Yu. Safarov and D. Vassiliev, The asymptotic distribution of eigenvalues of partial differential operators, A.M.S., Providence 1997.

[125] D. Scheglov, Complexity of typical triangle billiards, arXiv:1202.1244 (2012).

[126] K. Schmidt, Lectures on Cocycles of Ergodic Transformations Groups, Lecture Notes in Mathematics, N.1, MacMillan Co., India, 1977.

[127] R.E. Schwartz, Obtuse triangular billiards. I. Near the $(2,3,6)$ triangle, Experiment. Math. 15 (2006), 161 - 182.

[128] R.E. Schwartz, Obtuse triangular billiards. II. One hundred degrees worth of periodic trajectories, Experiment. Math. 18 (2009), 137 - 171.

[129] Ya. Sinai, On the foundations of the ergodic hypothesis for a dynamical system of statistical mechanics, Dokl. Akad. Nauk SSSR 153 (1963), 1261 - 1264. (In Russian. English transl: Soviet Math. Dokl. 4 (1963), 1818 - 1822.)

[130] Ya. Sinai, Dynamical systems with elastic reflections: Ergodic properties of dispersing billiards, Uspekhi Mat. Nauk 25 (1970), 141 - 192. (in Russian)

[131] J. Smillie, The dynamics of billiard flows in rational polygons, pp. 360 - 382 in Encyclopedia of Mathematical Sciences vol. 100, Springer-Verlag, New York 2000.

[132] A.B. Sossinsky, Configuration spaces of planar mechanical linkages with one degree of freedom, Russ. J. Math. Phys. 15 (2008), 530-541.

[133] P. Stäckel, Geodätische linien auf Polyederflächen, Rend. Circ. Mat. Palermo 22 (1906), $141-151$

[134] L. Stojanov, An estimate from above on the number of periodic orbits for semidispersed billiards, Comm. Math. Phys. 124 (1989), 217 - 227.

[135] L. Stojanov, Note on the periodic points of the billiard, J. Diff Geom. 34 (1991), 835 $-837$.

[136] D. Szasz, On the K-property of some planar hyperbolic billiards, Comm. Math. Phys. 145 (1992), 595 - 604.

[137] D. Szasz (editor), Hard Ball Systems and the Lorentz Gas, Encyclopedia of Mathematical Sciences vol. 101, Springer-Verlag, Berlin 2000.

[138] S. Tabachnikov, Billiards, Soc. Math. de France, Paris 1995.

[139] S. Tabachnikov, Geometry and billiards, American Mathematical Society, Providence, RI, 2005.

[140] S. Tabachnikov, Tire track geometry: variations on a theme, Israel J. Math. 151 (2006), $1-28$.

[141] S. Tabachnikov, Birkhoff billiards are insecure, Discrete Contin. Dyn. Syst. 23 (2009), 1035 - 1040.

[142] R. Trevino, On the ergodicity of flat surfaces of finite area, preprint (2012).

[143] W. Veech, Teichmuller curves in modular space, Eisenstein series, and an application to triangulat billiards, Inv. Math. 97 (1989), 553 - 583.

[144] W. Veech, Modular spaces of quadratic differentials, J. Analyse Math. 55 (1990), $117-171$.

[145] W. Veech, The billiard in a regular polygon, GAFA 2 (1992), 341 - 379.

[146] Ya. Vorobets, On the measure of the set of periodic points of a billiard, Mat. Notes 55 (1994), 455 - 460.

[147] Ya. Vorobets, Ergodicity of billiards in polygons, Mat. Sb. 188 (1997), 65 - 112.

[148] H. Weyl, Das asymptotische Verteilungsgesetz der Eigenwerte linearer partieller Differentialgleichungen (mit einer Anwendung auf die Theorie der Hohlraumstrahlung), Math. Ann. 71 (1912), 441 - 479. 
[149] M. Wojtkowski, Invariant families of cones and Lyapunov exponents, Erg. Theor. \& Dyn. Sys. 5 (1985), 145 - 161.

[150] M. Wojtkowski, Principles for the design of billiards with nonvanishing Lyapunov exponents, Comm. Math. Phys. 105 (1986), 391 - 414.

[151] M. Wojtkowski, Measure theoretic entropy of the system of hard spheres, Erg. Theor. \& Dyn. Sys. 8 (1988), 133 - 153.

[152] M. Wojtkowski, Two applications of Jacobi felds to the billiard ball problem, J. Diff. Geom. 40 (1994), 155 - 164.

[153] L.S. Young, Statistical properties of dynamical systems with some hyperbolicity, Ann. Math. 147 (1998), 585 - 650.

[154] T. Young, An essay on the cohesion of fluids, Philos. Trans. Roy. Soc. Lond. 95 (1805), $65-87$.

Nicolaus Copernicus University, Chopina 12/18, Torun 87-100; IM PAN, SniADECKICH 8, WARSZAWA 10, POLAND

E-mail address: gutkin@mat.umk.pl,gutkin@impan.pl 